\title{
Material similarity of scaled models
}

\author{
Shuai Wang ${ }^{\text {a,b,**}}$, Fei Xu ${ }^{\text {a,b,*, Xiaoyu Zhang }}{ }^{\text {a,b }}$, Leifeng Yang ${ }^{\text {a,b }}$ \\ ${ }^{\text {a }}$ School of Aeronautics, Northwestern Polytechnical University, Xi'an 710072, \\ Shaanxi, China; \\ ${ }^{\mathrm{b}}$ Institute for Computational Mechanics and Its Applications, Northwestern \\ Polytechnical University, Xi'an 710072, Shaanxi, China. \\ * Corresponding author, E-mail address: xufei@nwpu.edu.cn \\ ** E-mail address: wangsh2018@mail.nwpu.edu.cn \\ Authors ORCID: Shuai Wang, 0000-0003-0003-6209; Fei Xu, 0000-0003-1845- \\ 138X; Xiaoyu Zhang, 0000-0001-9760-3104.
}

\begin{abstract}
:
When different strain hardening and strain rate sensitive materials are used for scaled model and prototype, the traditional pure geometrical similarity laws of solid mechanics will fail. Although correcting the basic scaling factors of velocity, density and geometry have been developed to compensate for the material distortion in recent non-geometric scaling works, it is difficult to be widely used because of its inherent indirect (depending on the structural strain and strain rate responses) and inexact (having significant prediction errors for prototype) defects.

In this paper, a framework of material similarity, based on the new suggested material dimensionless numbers and the 'Material number vs. strain/strain-rate' function curves, are further developed, which represents the objective requirement of similarity theory for the basic mechanical properties of materials. It is demonstrated what is similitude materials of solid mechanics and how to use the best similitude materials to overcome the non-scalabilities of materials for identical or different materials. The direct and exact solution of the basic correction factors is further obtained and therefore overcomes the previous inherent indirect and inexact defects radically. Based on the similarity evaluation of different materials of the classical
\end{abstract}


constitutive models, the impacted structures of circular plate and crooked plate with strain hardening and strain rate sensitive materials are verified numerically. The results show the completely different materials can be exact similitude for various structural behaviors (strain, strain rate, stress and displacement) of time and space fields after using the best similitude materials; and the basic correction factors do not depend on the structural strain and strain rate responses. As a contrast, when the non-similitude materials are used, the similarity results are very sensitive to the selection of strain/strain-rate and often leads to failed predictions. In addition, for the material elastic and temperature effects, the proposed method is also discussed to be valid.

Keywords: Material number; Similarity; Scaling; Strain hardening; Strain rate; Temperature; Structural impact

\section{Introduction}

When the theoretical and numerical studies were practically impeded for structural impact phenomenon, verification by experiment is indispensable. As an important approach, the use of a scaled model instead of the full-size prototype has a great practical advantage $[1,2]$. The methodology with scaled model is termed as similarity, scaling or similitude [3]. In solid mechanics, scaling of the geometry, materials and external loads is the basic premise to establish similarity laws [3].

The traditional similarity laws were well-known and had been widely used in solid mechanics $[3,4]$. In these works, the identical materials were considered to simplify similarity difficulties of various material parameters, and the pure geometrical scaling was accomplished by a basic geometrically-similar factor to relate various physical quantities between prototype and scaled models. However, the traditional similarity laws were easily broken for the non-scalabilities such as size effects of material strain rate effects, gravity and fracture [3-8]. The non-scalability, that violating the traditional similarity laws, were usually said to be distorted [3].

The size effects of material strain-rate sensitivity were widespread [9], prompting researchers to develop the non-geometric scaling method with correction factors of external loads [10-15]. Oshiro and Alves [11] for example used a basic correction factor 
of impact velocity to compensate distortion of material strain rate effects. For simple analytical models [11] and complex numerical models [12], with the strain-rate sensitive mild steel, only had very small prediction errors. Similarly, the basic correction factor of mass/density for the impactor and structure were also developed in early work [13] and in recent work $[14,15]$. However, these developed methods were limited by an inherent defect that the basic correction factors were coupled to the structural average strain-rate responses and therefore were called to be indirect, which was hard to understand in similarity theory. To overcome this difficulty, a special power law constitutive equation was initially used to obtain the direct correction factor of velocity in the work of Oshiro and Alves [16]. When using simple analytical models, this direct method produced completely accurate similarity prediction for prototype. Nevertheless, it was only applicable to the particular power law form of the constitutive equation, not in all types.

Previous studies have assumed that a completely identical material was used for scaled model and prototype, and in fact, few experiments can satisfy this premise condition due to the absence cause of identical materials and the change of material properties in manufacturing [17]. In addition, impact problems usually involved many material viscous-plasticity (i.e., strain hardening and strain rate effects) parameters, so the use of different materials at scaling was an open problem. Based on the indirect correction technique, Alves and Oshiro [17] compensate distortion of the different materials in the quasi-static flow stress and the strain-rate sensitive effects. Although this method was precise for simple analytical models [17], it had been verified to have significant errors for complex problems of circular tubes axially impact [18]. Mazzariol, et al. $[19,20]$ further used the basic correction factor of density to compensate distortion of the different materials in density, and set the average strain-rate to $1 s^{-1}$ to simplify the previous indirect difficulties. Some different materials behaved good similarity, while others had significant errors [19]. A more comprehensive consideration for distortion of the different materials further including strain-hardening effects, temperature effects and failure are developed by Sadeghi et al. [21,22], and a fixed strain and strain-rate window instead of the average strain and strain-rate was used to 
simplify the previous indirect difficulty. However, this method can only provide inexact prediction ability and usually result in obvious errors for similarity. In further research, Wang and $\mathrm{Xu}$ et al. $[23,24]$ proposed geometric distortion similarity ability that adding a basic correction factor of geometrical thickness to compensate distortion of the different materials. But the average strain and average strain rate of the structure was required in the basic correction factor.

The inexact difficulty of the previous correction methods had caused some researchers to examine the influence of material parameters for the similarity. Wang and $\mathrm{Xu}$ et al. [25] qualitatively studied the influence of the material density, yield stress and strain-rate sensitive parameters for the optimal approximation similarity and found that the strain-rate sensitivity parameters play a decisive influence on the similarity errors. Davey et al. [26] studied influence of the Norton-Hoff constitutive parameters for similarity and therefore obtained more exact scaling feasibility for the complicated hot forging experimentation. In spite of this, these studies only focused on qualitative discussions for constitutive equation parameters in particular situations. For overcoming the previous indirect and inexact difficulties fundamentally, nor did they offer solutions.

A major criticism for all past studies is that they usually are limited by the indirect and inexact difficulties. As found in the research of our concerns, it is quite difficult to determine the response information of structural strain and strain rate in basic correction factors, especially for complex impacted structures; and none of these previous methods are precise enough to describe non-scalability of material distortion and they could even result in failed prediction in some cases. And in this paper, the main objective is to propose a complete framework of material similarity to overcome the two inherent difficulties of previous scaling approach.

In what follows, Section 2 introduces our newly material similarity framework in detail. Section 3 respectively investigated two scaled impacted models with strain hardening and strain-rate sensitive materials. Section 4 gives a discussion for extending the suggested method to more material mechanical properties and Section 5 summarize this work. 


\section{Material similarity framework}

\subsection{Previous scaling approach}

The similarity theory relating the prototype (p) and the scaled model (m) is wellknown and usually uses scaling factors of the physical quantities to conduct the forward scaling and the reverse prediction. For example, the basic geometrically-similar factor is usually defined as $\beta=\bar{L}_{m} / \bar{L}_{p}$ for all geometrical characteristic lengths $\bar{L}$.

For impact mechanics with strain hardening and strain-rate sensitive materials, recent researchers have developed several effective similarity systems [11,19,21-24] to derive scaling factors. As a more concise, clear and effective system, the density-lengthvelocity (DLV) dimensional analysis system [23] was expressed as the seven main dimensionless numbers

$$
\begin{aligned}
& \Pi_{\sigma}=\left[\frac{\rho V^{2}}{\sigma}\right], \Pi_{\varepsilon}=[\varepsilon], \Pi_{\dot{\varepsilon}}=\left[\frac{\dot{\varepsilon} \bar{L}}{V}\right], \Pi_{t}=\left[\frac{t V}{\bar{L}}\right], \\
& \Pi_{\delta}=\left[\frac{\delta}{\bar{L}}\right], \Pi_{G}=\left[\frac{\bar{m}}{\rho \bar{L}^{3}}\right], \Pi_{F}=\left[\frac{\rho \bar{L}^{2} V^{2}}{F}\right] .
\end{aligned}
$$

where $\rho, V, \sigma, \varepsilon, \dot{\varepsilon}, t, \delta, \bar{m}$ and $F$ are density, velocity, stress, strain, strain rate, time, displacement, mass and force, respectively, that each physical quantity is related by the three bases of the density $\rho$, the length $\bar{L}$ and the velocity $V$.

The well-known similarity theory requires that all predominant dimensionless numbers of a scaled model must be equal to those of the prototype [3,4], i.e. $\left(\Pi_{i}\right)_{m}=$ $\left(\Pi_{i}\right)_{p}$. When the seven numbers are used, main scaling factors can be directly obtained as Table 1 , in which $\beta, \beta_{V}$ and $\beta_{\rho}$ are three independent basic factors.

Table 1 Main scaling factors of impact mechanics.

\begin{tabular}{llll}
\hline Variable & Scaling factor & Variable & Scaling factor \\
\hline Length, $\bar{L}$ & $\beta=\bar{L}_{m} / \bar{L}_{p}$ & Strain rate, $\dot{\varepsilon}$ & $\beta_{\dot{\varepsilon}}=\beta_{V} / \beta$ \\
Density, $\rho$ & $\beta_{\rho}=\rho_{m} / \rho_{p}$ & Time, $t$ & $\beta_{t}=\beta / \beta_{V}$ \\
Velocity, $V$ & $\beta_{V}=V_{m} / V_{p}$ & Displacement, $\delta$ & $\beta_{\delta}=\beta$ \\
Stress, $\sigma$ & $\beta_{\sigma}=\beta_{\rho} \beta_{V}^{2}$ & Mass, $\bar{m}$ & $\beta_{\bar{m}}=\beta_{\rho} \beta^{3}$
\end{tabular}


Strain, $\varepsilon \quad \beta_{\varepsilon}=1 \quad$ Force, $F \quad \beta_{F}=\beta_{\rho} \beta^{2} \beta_{V}^{2}$

To compensate for the non-scalability of material strain hardening and strain-rate sensitive effects, the similarity theory should respect the viscous-plasticity constitutive equation,

$$
\sigma_{d}=\sigma_{0} f(\varepsilon, \dot{\varepsilon})
$$

where $\sigma_{0}$ and $\sigma_{d}$ are the quasi-static and dynamic flow stress, respectively.

When combining the scaling relations $\beta_{\sigma}=\beta_{\rho} \beta_{V}^{2}, \beta_{\varepsilon}=1, \beta_{\dot{\varepsilon}}=\beta_{V} / \beta$ and Eq. (2), the correction factor of velocity is derived as

$$
\beta_{V}=\sqrt{\frac{\beta_{\sigma_{d}}}{\beta_{\rho}}}=\sqrt{\frac{\beta_{\sigma_{0}}}{\beta_{\rho}} \frac{f_{m}\left(\beta_{\varepsilon} \varepsilon_{p}, \beta_{\dot{\varepsilon}} \dot{\varepsilon}_{p}\right)}{f_{p}\left(\varepsilon_{p}, \dot{\varepsilon}_{p}\right)}}=\sqrt{\frac{\beta_{\sigma_{0}}}{\beta_{\rho}} \frac{f_{m}\left(\varepsilon_{p}, \dot{\varepsilon}_{p} \beta_{V} / \beta\right)}{f_{p}\left(\varepsilon_{p}, \dot{\varepsilon}_{p}\right)}}
$$

which can be solved by a numerical method if the structural responses of the strain $\varepsilon_{p}$ and the strain rate $\dot{\varepsilon}_{p}$ are known.

Similar studies in previous works also define the correction factor of density and the geometry (for geometrically-distorted scaling). Since the three correction methods can convert to each other $[23,24]$, the velocity correction factor Eq. (3), as one of the typical representatives, is mainly discussed in this paper.

In the above correction procedure, understanding Eq. (3) is crucial. As mentioned in Section 1, two main inherent difficulties, existing in all recent developed approaches, can be found as follows.

- Non-direct: the velocity correction factor is coupled to the structural response of strain and strain-rate, and therefore it is difficult obtained directly. This non-direct phenomenon has not been seen in other known scaling laws.

- Inaccuracy: the dynamic strain and strain rate responses of structure in time and space fields lead to different values of $\beta_{V}$, while the similarity theory requires $\beta_{V}$ being constant. To overcome this problem, the previous works usually used the average strain $\bar{\varepsilon}_{p}$ and the average strain rate $\overline{\dot{\varepsilon}}_{p}$ (or some particular values, etc.) to obtain an approximate value of $\beta_{V}$. Thus, the exact similarity does not exist.

Therefore, it seems worthwhile to expand the previous methodologies by further 
overcoming the non-direct and inaccurate difficulty.

\subsection{Dimensionless numbers of material similarity}

To derive the essential similarity criterion for strain hardening and strain-rate sensitive materials, in what follows, we further investigate similarity of the material second-order effects by the slope of the most basic stress - strain/strain-rate relation.

Without loss of generality, the total differential relation (or one-dimensional incremental relation of plasticity) of the constitutive equation Eq. (2) is expressed as

$$
d \sigma_{d}=\sigma_{0} \frac{\partial f(\varepsilon, \dot{\varepsilon})}{\partial \varepsilon} d \varepsilon+\sigma_{0} \frac{\partial f(\varepsilon, \dot{\varepsilon})}{\partial \dot{\varepsilon}} d \dot{\varepsilon}=E_{t}(\varepsilon, \dot{\varepsilon}) d \varepsilon+K_{t}(\varepsilon, \dot{\varepsilon}) d \dot{\varepsilon},
$$

where $E_{t}(\varepsilon, \dot{\varepsilon})=\sigma_{0} \partial f(\varepsilon, \dot{\varepsilon}) / \partial \varepsilon$ and $K_{t}(\varepsilon, \dot{\varepsilon})=\sigma_{0} \partial f(\varepsilon, \dot{\varepsilon}) / \partial \dot{\varepsilon}$ are material tangent modulus. Apparently, besides the dynamic flow stress $\sigma_{d}$, the important material parameters $E_{t}$ and $K_{t}$ for strain hardening and strain-rate sensitive effects appear.

For two different materials, Eq. (4) can be written as

$$
d\left(\sigma_{d}\right)_{m}=\left(E_{t}\right)_{m} d \varepsilon_{m}+\left(K_{t}\right)_{m} d \dot{\varepsilon}_{m}
$$

and

$$
d\left(\sigma_{d}\right)_{p}=\left(E_{t}\right)_{p} d \varepsilon_{p}+\left(K_{t}\right)_{p} d \dot{\varepsilon}_{p}
$$

respectively.

Substitution $\left(\sigma_{d}\right)_{m}=\beta_{\sigma_{d}}\left(\sigma_{d}\right)_{p},\left(E_{t}\right)_{m}=\beta_{E_{t}}\left(E_{t}\right)_{p},\left(K_{t}\right)_{m}=\beta_{K_{t}}\left(K_{t}\right)_{p}, \varepsilon_{m}=$ $\beta_{\varepsilon} \varepsilon_{p}$ and $\dot{\varepsilon}_{m}=\beta_{\dot{\varepsilon}} \dot{\varepsilon}_{p}$ into Eq. (5a) leads to

$$
d \beta_{\sigma_{d}}\left(\sigma_{d}\right)_{p}=\beta_{E_{t}}\left(E_{t}\right)_{p} d\left(\beta_{\varepsilon} \varepsilon_{p}\right)+\beta_{K_{t}}\left(K_{t}\right)_{p} d\left(\beta_{\dot{\varepsilon}} \dot{\varepsilon}_{p}\right)
$$

It can be rewritten as

$$
d\left(\sigma_{d}\right)_{p}=\frac{\beta_{E_{t}} \beta_{\varepsilon}}{\beta_{\sigma_{d}}}\left(E_{t}\right)_{p} d \varepsilon_{p}+\frac{\beta_{K_{t}} \beta_{\dot{\varepsilon}}}{\beta_{\sigma_{d}}}\left(K_{t}\right)_{p} d \dot{\varepsilon}_{p},
$$

For similarity, Eq. (6b) and Eq. (5b) should be identical, which should respect the quantitative similarity criterion

$$
\frac{\beta_{E_{t}} \beta_{\varepsilon}}{\beta_{\sigma_{d}}}=1
$$


and

$$
\frac{\beta_{K_{t}} \beta_{\dot{\varepsilon}}}{\beta_{\sigma_{d}}}=1
$$

The two criterions can also be re-written as the dimensionless equations as

$$
\left(\Pi_{E_{t}}\right)_{m}=\left(\Pi_{E_{t}}\right)_{p}
$$

and

$$
\left(\Pi_{K_{t}}\right)_{m}=\left(\Pi_{K_{t}}\right)_{p}
$$

respectively, where $\Pi_{E_{t}}=\left[\frac{E_{t} \varepsilon}{\sigma_{d}}\right]$ and $\Pi_{K_{t}}=\left[\frac{k_{t} \dot{\varepsilon}}{\sigma_{d}}\right]$ are dimensionless numbers of the material strain hardening and strain-rate sensitive effects, respectively.

It is easy to see that the numbers $\Pi_{E_{t}}$ and $\Pi_{K_{t}}$ are the dominant dimensionless parameters for material similarity. For convenience, the two dimensionless numbers are termed as "material similarity number / material number $(M n)$ " by the present paper and are further expressed as

$$
\operatorname{Mn}\{\varepsilon\}=\frac{E_{t}(\varepsilon, \dot{\varepsilon}) \varepsilon}{\sigma_{d}}=\frac{(\partial f(\varepsilon, \dot{\varepsilon}) / \partial \varepsilon) \varepsilon}{f(\varepsilon, \dot{\varepsilon})}=\frac{\partial \ln f(\varepsilon, \dot{\varepsilon})}{\partial \ln \varepsilon}
$$

and

$$
\operatorname{Mn}\{\dot{\varepsilon}\}=\frac{K_{t}(\varepsilon, \dot{\varepsilon}) \dot{\varepsilon}}{\sigma_{d}}=\frac{(\partial f(\varepsilon, \dot{\varepsilon}) / \partial \dot{\varepsilon}) \dot{\varepsilon}}{f(\varepsilon, \dot{\varepsilon})}=\frac{\partial \ln f(\varepsilon, \dot{\varepsilon})}{\partial \ln \dot{\varepsilon}}, 1
$$

where the curly braces $\{\varepsilon\}$ and $\{\dot{\varepsilon}\}$ represent associations with the material strain hardening and strain-rate sensitive effects, respectively.

From Eq. (9) follows that the material number can be considered as the ratio of a

${ }^{1}$ For the material number $\operatorname{Mn}\{\dot{\varepsilon}\}$, the similar forms $\lambda=[\partial \sigma / \partial \ln \dot{\varepsilon}],[\partial \tau / \partial \ln \dot{\gamma}]$, $\left[\partial \tau_{\text {eff }} / \partial \ln \dot{\gamma}\right]$ and $\left[\partial \sigma_{d} / \partial \ln \dot{\varepsilon}\right][27]$, termed as the strain-rate sensitivity coefficient (or exponent), were used for macroscopic dynamic plasticity and dislocation dynamic studies, where $\tau, \dot{\gamma}$ and $\tau_{\text {eff }}$ were shear stress, shear strain-rate and effective shear stress, respectively. It is noted that the first three forms of $\lambda$ have the dimension $M P a$; while the last form is a dimensionless number and equivalent to $\operatorname{Mn}\{\dot{\varepsilon}\}$. 
material's ability to maintain inertial motion deformation (i.e., $E_{t} \varepsilon$ at space and $K_{t} \dot{\varepsilon}$ at time) to its own resistance (i.e., $\sigma_{d}$ ); it can also be considered as the rate of change of the logarithmic exponent of dynamic flow stress (i.e., $n=\ln f(\varepsilon, \dot{\varepsilon})$ with $n$ being exponent of the natural constant $e$ ) relative to the logarithmic exponent of deformation (i.e., $\ln \varepsilon$ at space and $\ln \dot{\varepsilon}$ at time); it can be also understood as the intrinsic requirement of similarity to material basic mechanical properties of strain hardening and strain-rate sensitive effects.

The above derivation indicates that the proposed material numbers control similarity of the material strain hardening and strain-rate sensitive effects, and therefore they must be respected to perform scaled experimentation in solid mechanics.

\subsection{Direct and exact solution of correction factor}

In an attempt to solve the inherent non-direct and inaccuracy difficulty of previous scaling methods, the material similarity criterion developed in Section 2.2 is applied.

The similarity criterion $M n_{m}\left\{\varepsilon_{m}\right\}=M n_{p}\left\{\varepsilon_{p}\right\}$ (Eq. (8a)) leads to

$$
\begin{aligned}
& \frac{\partial \ln f_{m}\left(\varepsilon_{m}, \dot{\varepsilon}_{m}\right)}{\partial \ln \varepsilon_{m}}=\frac{\partial \ln f_{m}\left(\beta_{\varepsilon} \varepsilon_{p}, \beta_{\dot{\varepsilon}} \dot{\varepsilon}_{p}\right)}{\partial \ln \left(\beta_{\varepsilon} \varepsilon_{p}\right)}=\frac{\partial \ln f_{m}\left(\beta_{\varepsilon} \varepsilon_{p}, \beta_{\dot{\varepsilon}} \dot{\varepsilon}_{p}\right)}{\partial \ln \varepsilon_{p}} \\
& =\frac{\partial \ln f_{p}\left(\varepsilon_{p}, \dot{\varepsilon}_{p}\right)}{\partial \ln \varepsilon_{p}}
\end{aligned}
$$

Let's assume that $\dot{\varepsilon}_{p}$ is some arbitrary constant $\dot{\varepsilon}_{C}$, Eq. (10) becomes

$$
d \ln f_{m}\left(\beta_{\varepsilon} \varepsilon_{p}, \beta_{\dot{\varepsilon}} \dot{\varepsilon}_{C}\right)=d \ln f_{p}\left(\varepsilon_{p}, \dot{\varepsilon}_{C}\right)
$$

So, the integral of Eq. (11) results in

$$
\frac{f_{m}\left(\beta_{\varepsilon} \varepsilon_{p}, \beta_{\dot{\varepsilon}} \dot{\varepsilon}_{C}\right)}{f_{p}\left(\varepsilon_{p}, \dot{\varepsilon}_{C}\right)}=\mathrm{constant}
$$

In a similar way, when $\varepsilon_{p}$ is some arbitrary constant $\varepsilon_{C}$, the similarity criterion $M n_{m}\left\{\dot{\varepsilon}_{m}\right\}=M n_{p}\left\{\dot{\varepsilon}_{p}\right\}$ (Eq. (8b)) leads to

$$
\frac{f_{m}\left(\beta_{\varepsilon} \varepsilon_{C}, \beta_{\dot{\varepsilon}} \dot{\varepsilon}_{p}\right)}{f_{p}\left(\varepsilon_{C}, \dot{\varepsilon}_{p}\right)}=\mathrm{constant}
$$

The combination of Eq. (12a) and Eq. (12b) signifies 


$$
\frac{f_{m}\left(\beta_{\varepsilon} \varepsilon_{p}, \beta_{\dot{\varepsilon}} \dot{\varepsilon}_{p}\right)}{f_{p}\left(\varepsilon_{p}, \dot{\varepsilon}_{p}\right)} \equiv \mathrm{constant} \text { if }\left\{\begin{array}{l}
M n_{m}\left\{\varepsilon_{m}\right\}=M n_{p}\left\{\varepsilon_{p}\right\} \\
M n_{m}\left\{\dot{\varepsilon}_{m}\right\}=M n_{p}\left\{\dot{\varepsilon}_{p}\right\}
\end{array}\right.
$$

Then, the direct and exact solution for Eq. (3) can be obtained as

$$
\beta_{V}=\sqrt{\frac{\beta_{\sigma_{0}}}{\beta_{\rho}} \frac{f_{m}\left(\varepsilon_{p}, \dot{\varepsilon}_{p} \beta_{V} / \beta\right)}{f_{p}\left(\varepsilon_{p}, \dot{\varepsilon}_{p}\right)}} \equiv \text { constant if }\left\{\begin{array}{l}
M n_{m}\left\{\varepsilon_{m}\right\}=M n_{p}\left\{\varepsilon_{p}\right\} \\
M n_{m}\left\{\dot{\varepsilon}_{m}\right\}=M n_{p}\left\{\dot{\varepsilon}_{p}\right\}
\end{array}\right.
$$

in which $\varepsilon_{p}$ and $\dot{\varepsilon}_{p}$ can be arbitrary values and have no effect on the solution (in other words, it can be eliminated from the equation). To illustrate the solution explicitly, an example of constitutive equation is presented in Appendix A.

In most cases, it is difficult for two different materials to accurately satisfy the similarity criterion $M n_{m}\left\{\varepsilon_{m}\right\}=M n_{p}\left\{\varepsilon_{p}\right\}$ and $M n_{m}\left\{\dot{\varepsilon}_{m}\right\}=M n_{p}\left\{\dot{\varepsilon}_{p}\right\}$. Therefore, by allowing a certain degree of errors $\kappa_{1}$ and $\kappa_{2}$, they are relaxed as

$$
|\Delta M n\{\varepsilon\}|=\left|M n_{m}\left\{\varepsilon_{m}\right\}-M n_{p}\left\{\varepsilon_{p}\right\}\right| \leq \kappa_{1}
$$

and

$$
|\Delta M n\{\dot{\varepsilon}\}|=\left|M n_{m}\left\{\dot{\varepsilon}_{m}\right\}-M n_{p}\left\{\dot{\varepsilon}_{p}\right\}\right| \leq \kappa_{2},
$$

respectively.

And the direct and exact solution of Eq. (14) is further relaxed as

$$
\beta_{V}=\sqrt{\frac{\beta_{\sigma_{0}}}{\beta_{\rho}} \frac{f_{m}\left(\hat{\varepsilon}_{p}, \hat{\dot{\varepsilon}}_{p} \beta_{V} / \beta\right)}{f_{p}\left(\widehat{\varepsilon}_{p}, \hat{\dot{\varepsilon}}_{p}\right)}} \approx \text { constant if }\left\{\begin{array}{l}
|\Delta M n\{\varepsilon\}| \leq \kappa_{1} \\
|\Delta M n\{\dot{\varepsilon}\}| \leq \kappa_{2}
\end{array},\right.
$$

where $\hat{\varepsilon}_{p}$ and $\hat{\varepsilon}_{p}$ can be some order of magnitude of structural strain and strain rate responses, easily obtained through very simple estimation or engineering experience.

\subsection{Using material similarity curves to design scaled model}

The above research proves that the key to obtain the direct and exact solution of basic correction factor is to select the best similitude materials. To evaluate the material similarity more intuitively and conveniently, Eq. (15a) and (15b) (using $\beta_{\varepsilon}=1$ and $\beta_{\dot{\varepsilon}}=\beta_{V} / \beta$, respectively) are plotted in Fig. 1, in which the 'material number - 
strain/strain-rate' curves are termed as 'material similarity curves' by the present paper.

- For strain hardening effects, since the strain $\varepsilon_{m}$ is the same as $\varepsilon_{p}$, the best similitude material for scaled model is a material whose $\operatorname{Mn}\{\varepsilon\}$ at $\varepsilon_{p}$ is basically identical with the corresponding prototype $\operatorname{Mn}\{\varepsilon\}$ at $\varepsilon_{p}$, Fig. 1a.

- For the strain-rate sensitive effects, since $\dot{\varepsilon}_{m}$ is proportional to $\dot{\varepsilon}_{p}$, the best similitude material for scaled model is a material whose $\operatorname{Mn}\{\dot{\varepsilon}\}$ at $\left(\beta_{\dot{\varepsilon}} \dot{\varepsilon}_{p}\right)$ is basically identical with the corresponding prototype $\operatorname{Mn}\{\dot{\varepsilon}\}$ at $\dot{\varepsilon}_{p}$, Fig. 1b. For convenience, $\beta_{\dot{\varepsilon}}$ in Fig. $1 \mathrm{~b}$ can be taken to be $\tilde{\beta}_{\dot{\varepsilon}}=\beta_{V} / \beta \approx \sqrt{\beta_{\sigma_{0}} / \beta_{\rho}} / \beta$ (it does not require iteration) when $\beta_{V}$ is approximated as $\sqrt{\beta_{\sigma_{0}} / \beta_{\rho}}$ from Eq. (3).

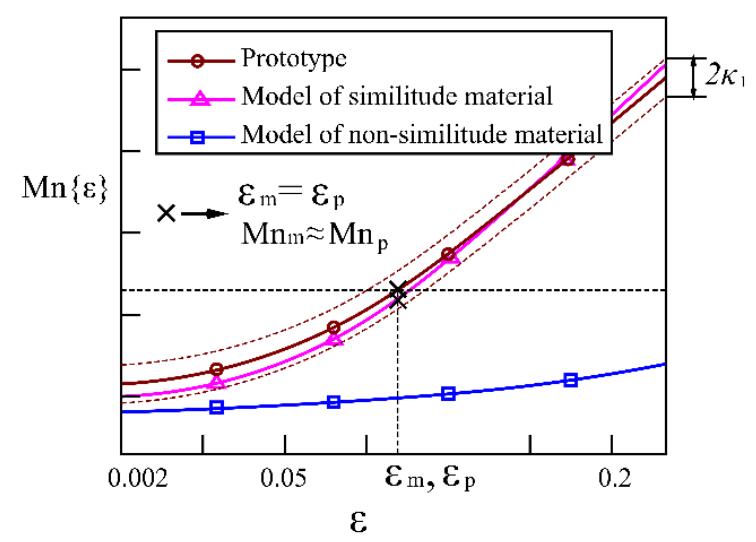

(a) Mn vs. Strain

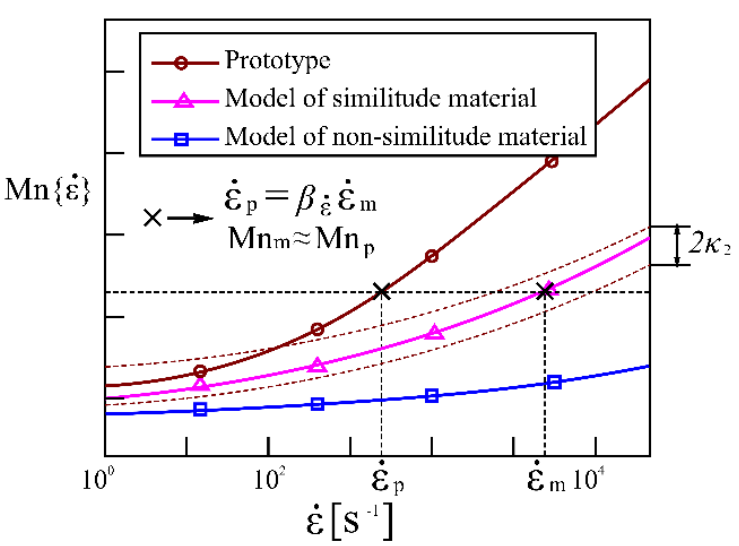

(b) Mn vs. Strain-rate

Fig. 1 Illustration the material similarity criterion (Eq. (15a) and (15b)).

\section{Verification}

In this section, two impacted structures with strain hardening and strain-rate sensitive materials are used to validate the proposed methods.

\subsection{Impacted circular plate with strain hardening materials}

To verify material similarity of the strain hardening effects, the simple impacted structure of a clamped circular plate subjected to impulsive velocity (as shown in Fig. 2 ) is used, and the well-known parabolic hardening relation [28]

$$
\sigma_{d}=\sigma_{0}+k \varepsilon^{\mathrm{n}}
$$

with $k$ and $n$ being material constants is considered. 


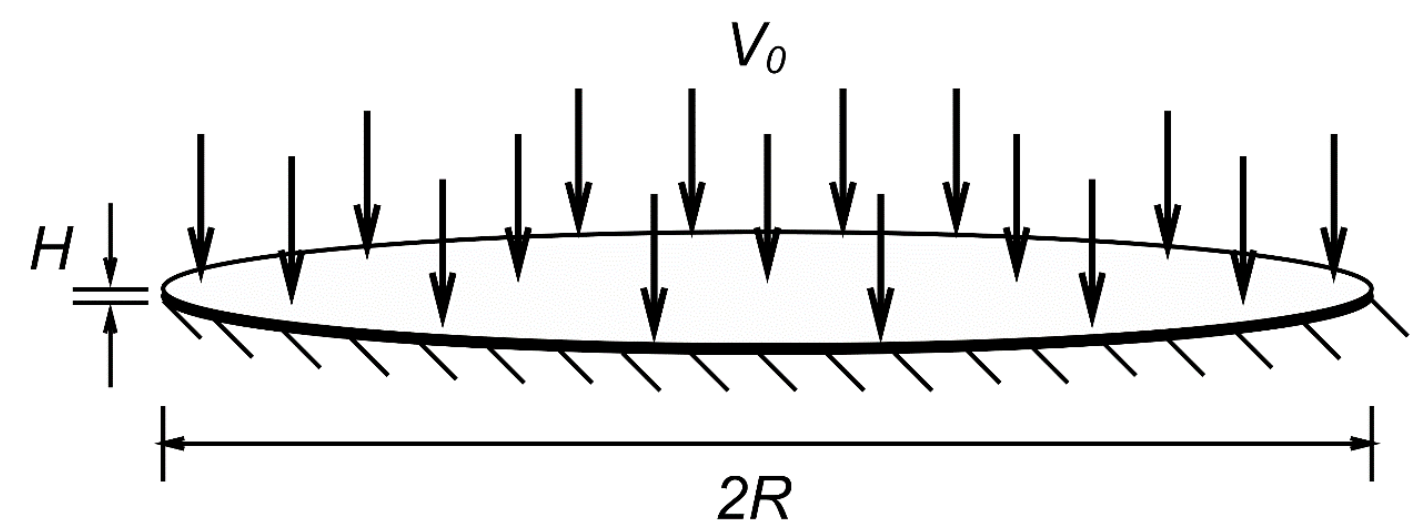

Fig. 2 A clamped circular plate with $R=300$ and $H=4$ is subjected to the impulsive velocity $V_{0}=60 \mathrm{~m} / \mathrm{s}$.

The substitution of $E_{t}(\varepsilon)=\partial \sigma_{d} / \partial \varepsilon=k n \varepsilon^{n-1}$ into Eq. (9a) obtains the material number

$$
\operatorname{Mn}\{\varepsilon\}=\frac{E_{t} \varepsilon}{\sigma_{d}}=n \frac{k \varepsilon^{n}}{\sigma_{0}+k \varepsilon^{n}} .
$$

Then, the material similarity is evaluated in Fig. 3 with eight different materials (referring to Table B.1). Apparently, if the factor $\beta_{\varepsilon}=1$ is used to scale strain, the OFHC copper and the Nickel 200, the 1006 steel and the $\alpha$ - titanium alloy as well as the S-7 Tool Steel and the Tungsten Alloy can be intuitively considered as the best similitude materials. 


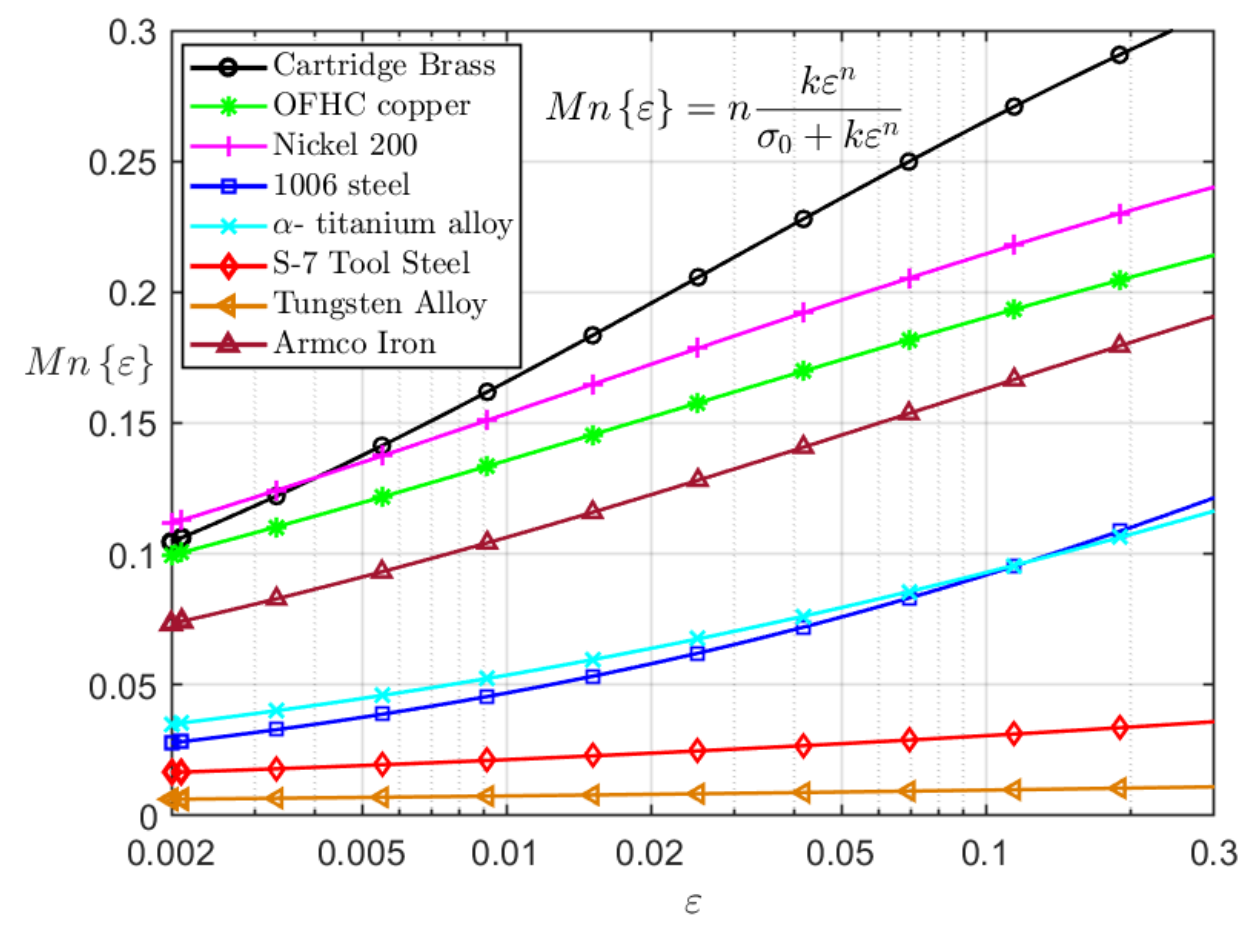

Fig. 3 Material similarity curves of the parabolic hardening equation Eq. (17).

\section{- 3.1.1 Formulation}

For prototype, the geometry and loads are exactly the same as the impacted circular plate. And the Nickel 200 is used. For scaled models, all geometric sizes are scaled by $\beta=1 / 100$. The impact velocity is scaled by $\beta_{V}$ (Eq. (16)). The best similitude material, OFHC copper, and the non-similitude material (as a contrast), Tungsten Alloy, are selected intuitively, according to Fig. 3. Furthermore, the quantitative evaluation for material similarity is carried out by Eq. (15a) in which $\kappa_{1}$ is assumed to 0.03 that being about one tenth of the maximum vertical coordinate of Fig. 3. In addition, the five orders of magnitude of characteristic strain, $\hat{\varepsilon}_{p}=$ $0.005,0.01,0.05,0.1,0.2$ are used to Eq. (16), respectively. Ten kinds of cases with the evaluation of material similarity as well as the basic scaling factors $\beta, \beta_{\rho}$ and $\beta_{V}$ are listed in Table 2. From $\hat{\varepsilon}_{p}=0.005$ to $\hat{\varepsilon}_{p}=0.2$, the factor $\beta_{V}$ for the nonsimilitude material Tungsten changed by 28 percent; while, for the similitude material Copper, it only changed by 4 percent.

Table 2 Material similarity evaluation and three basic scaling factors.

\begin{tabular}{lllllllll}
\hline Scaled model & Material & $\beta_{\varepsilon}$ & $\hat{\varepsilon}_{m}$ & $|\Delta M n|$ & $\beta$ & $\beta_{\rho}$ & $\beta_{V}$ \\
\hline
\end{tabular}




\begin{tabular}{llllllll}
\hline $1\left(\hat{\varepsilon}_{p}=0.005\right)$ & Tungsten & 1 & 0.005 & 0.128 & 0.01 & 1.910 & 1.743 \\
$2\left(\hat{\varepsilon}_{p}=0.01\right)$ & Tungsten & 1 & 0.01 & 0.146 & 0.01 & 1.910 & 1.662 \\
$3\left(\hat{\varepsilon}_{p}=0.05\right)$ & Tungsten & 1 & 0.05 & 0.188 & 0.01 & 1.910 & 1.453 \\
$4\left(\hat{\varepsilon}_{p}=0.1\right)$ & Tungsten & 1 & 0.1 & 0.205 & 0.01 & 1.910 & 1.357 \\
$5\left(\hat{\varepsilon}_{p}=0.2\right)$ & Tungsten & 1 & 0.2 & 0.221 & 0.01 & 1.910 & 1.261 \\
$6\left(\hat{\varepsilon}_{p}=0.005\right)$ & Copper & 1 & 0.005 & 0.015 & 0.01 & 1.007 & 0.726 \\
$7\left(\hat{\varepsilon}_{p}=0.01\right)$ & Copper & 1 & 0.01 & 0.018 & 0.01 & 1.007 & 0.722 \\
$8\left(\hat{\varepsilon}_{p}=0.05\right)$ & Copper & 1 & 0.05 & 0.023 & 0.01 & 1.007 & 0.711 \\
$9\left(\hat{\varepsilon}_{p}=0.1\right)$ & Copper & 1 & 0.1 & 0.024 & 0.01 & 1.007 & 0.705 \\
$10\left(\hat{\varepsilon}_{p}=0.2\right)$ & Copper & 1 & 0.2 & 0.026 & 0.01 & 1.007 & 0.699 \\
\hline
\end{tabular}

- 3.1.2 Results

The clamped circular plates are modelled in the ABAQUS software using the CAX4R axisymmetric elements and discretized with 600 elements in radius and 8 in thickness direction. In order to eliminate the errors of elasticity for similarity as much as possible, the elastic modulus is set to 10000 times of the quasi-static flow stress so that the maximum elastic strain is limited to 0.0001 . The various responses of the center point and neutral surface of the circular plates are analyzed below.

The refined evaluation of similarity by the strain and stress responses in time and space fields is plotted in Fig. 4. For the scaled models with the non-similitude material Tungsten, the strain and stress responses of these models deviate significantly from the prototype responses whether in the time field or in the space field, Fig. $4 \mathrm{a}$, c, e and g. And the predicted results are very sensitive to the characteristic strain used in Eq. (16). While, for the scaled models with the best similitude material Copper, the strain and stress responses of scale model is almost exactly consistent with those of the prototype, Fig. $4 \mathrm{~b}, \mathrm{~d}$, f and $\mathrm{h}$. And the predicted results are not affected by the characteristic strain. 

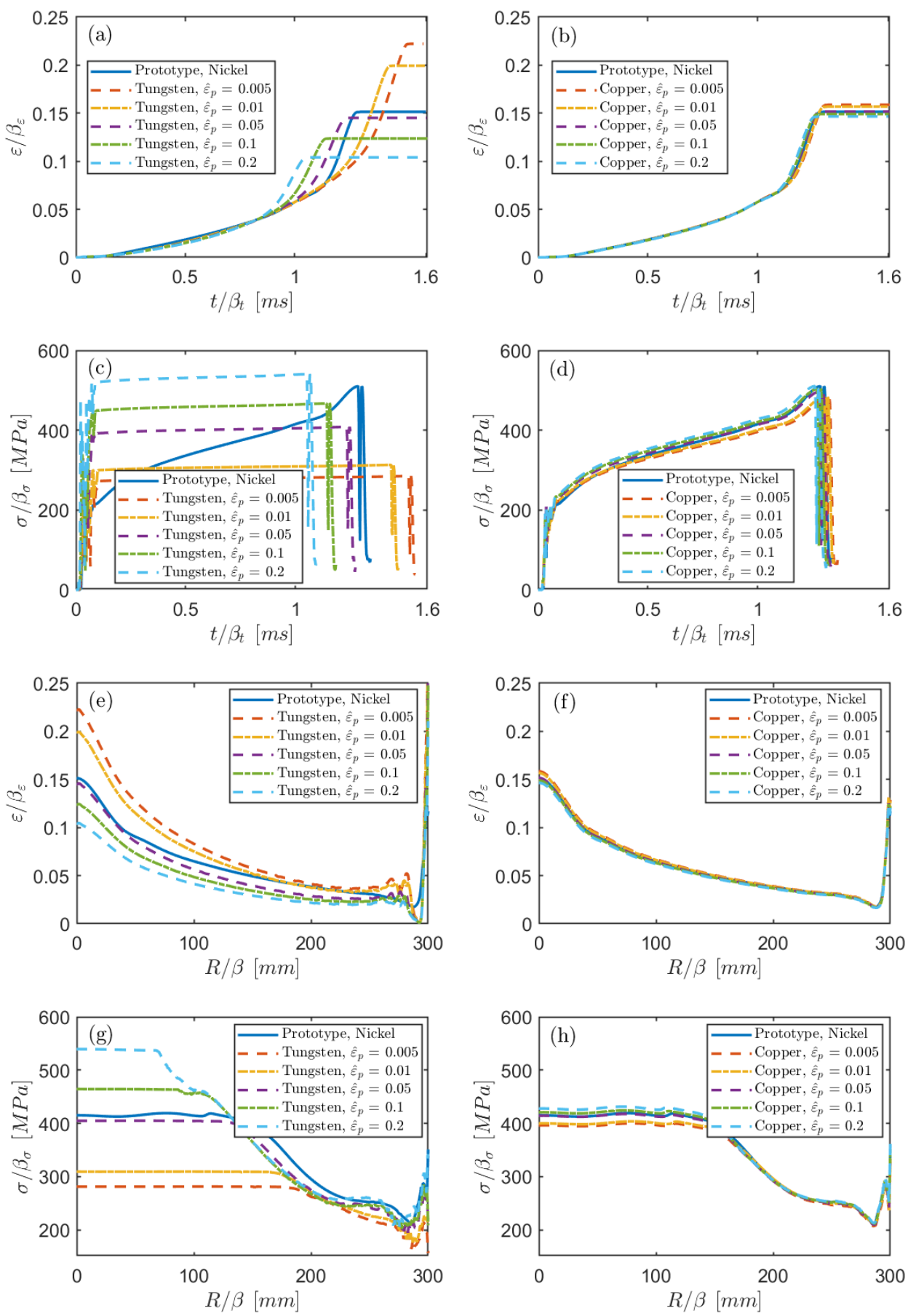

Fig. 4 Strain and stress response for scaled impacted circular plates with the similitude material Tungsten and the non-similitude material Copper.

Further evaluation of similarity by the displacement responses in time and space fields is shown in Fig. 5. The responses of the non-similitude material Tungsten are very sensitive to the characteristic strain $\hat{\varepsilon}_{p}$ and lead to significant predicted errors (maximum error being 19 percent) for prototype, Fig. 5a and c. While, the best similitude material Copper responses are always nearly the same as the prototype 
responses, only with the maximum error 0.2 percent, Fig. $5 \mathrm{~b}$ and $\mathrm{d}$.
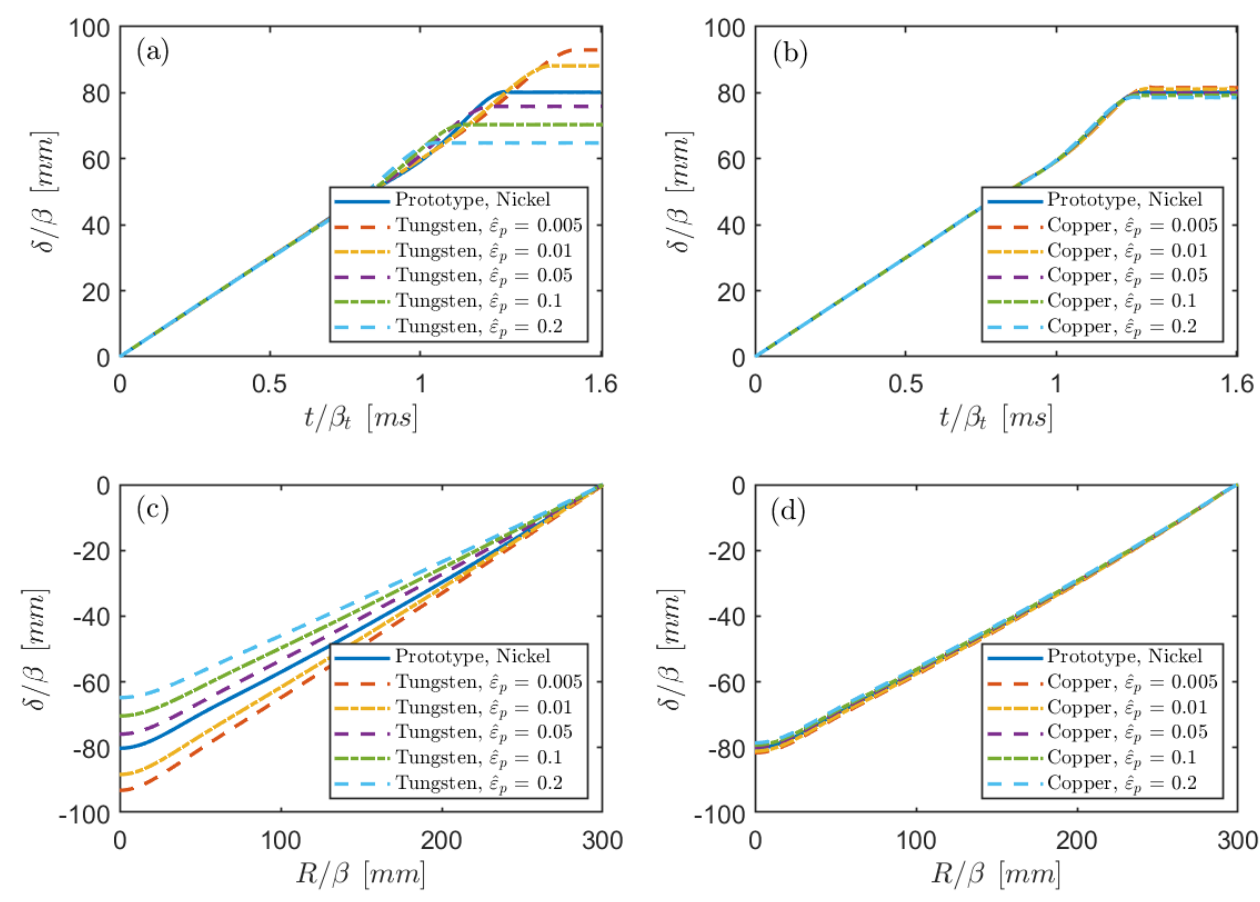

Fig. 5 Displacement responses for scaled impacted circular plates with the similitude material Tungsten and the non-similitude material Copper.

\subsection{Impacted circular plate with strain-rate sensitive materials}

To verify material similarity of the strain-rate sensitive effects, the impacted circular plate model in Fig. 2 is used again, and the Cowper-Symonds equation [3]

$$
\sigma_{d}=\sigma_{0}\left[1+\left(\frac{\dot{\varepsilon}}{D}\right)^{1 / \mathrm{P}}\right],
$$

with material parameters $D$ and $P$ is considered.

The substitution of $K_{t}(\dot{\varepsilon})=\partial \sigma_{d} / \partial \dot{\varepsilon}=\left(\sigma_{0} / P D\right)(\dot{\varepsilon} / D)^{1 / P-1}$ into Eq. (9b) obtains the material number

$$
\operatorname{Mn}\{\dot{\varepsilon}\}=\frac{K_{t} \dot{\varepsilon}}{\sigma_{d}}=\frac{1}{P} \frac{(\dot{\varepsilon} / D)^{1 / \mathrm{P}}}{1+(\dot{\varepsilon} / D)^{1 / \mathrm{P}}} .
$$

Then, the material similarity of strain-rate sensitive effects is evaluated in Fig. 6 with eight different materials in Table B.2. Apparently, if the factor $\beta_{\dot{\varepsilon}}=1$ (i.e., the geometry does not scale) is used to scale strain-rate, the S355 steel and the Highstrength steel S690, the Aluminum 6061-T6 and the Copper as well as the Dual Phase 
1200 steel and the Stainless steel 304 can be intuitively considered as the best similitude materials.

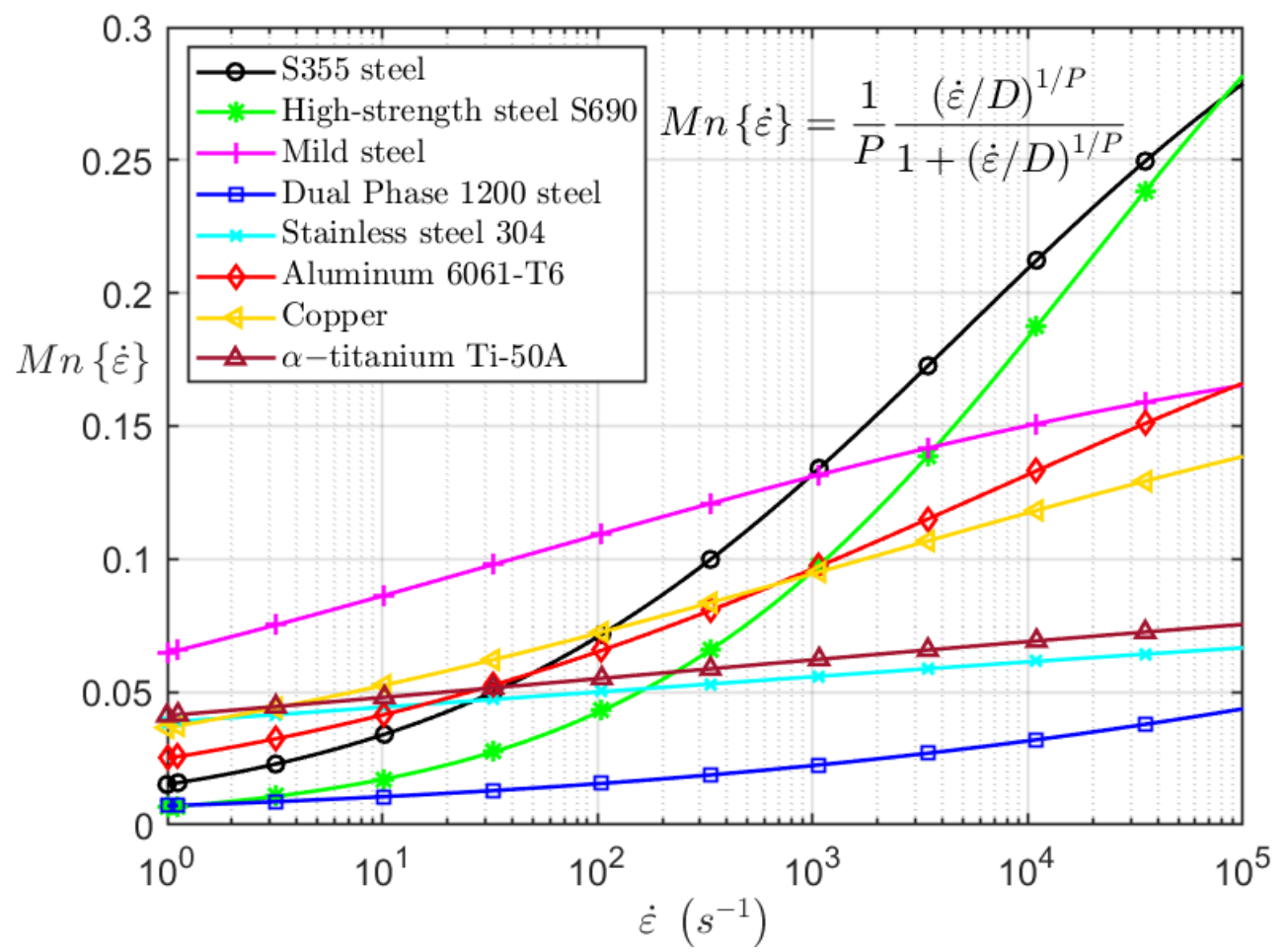

Fig. 6 Material similarity curves of the Cowper-Symonds equation Eq. (19).

\section{- 3.2.1 Formulation}

The impacted circular plates in Fig. 2 is again considered as the prototype. And the High-strength steel S690 is used. For scaled models, all geometric sizes are scaled by $\beta=1 / 100$. The impact velocity is scaled by $\beta_{V}$ (Eq. (16)). Assume the characteristic strain rate of prototype $\hat{\dot{\varepsilon}}_{p} \in\left[0,400 \mathrm{~s}^{-1}\right]$, the best similitude material, Dual Phase 1200 steel (DP1200), and the non-similitude material (as a contrast), S355 steel, are selected intuitively, according to Fig. $1 \mathrm{~b}$ with $\tilde{\beta}_{\dot{\varepsilon}} \approx \sqrt{\beta_{\sigma_{0}} / \beta_{\rho}} / \beta=78$ for the S355 and $\tilde{\beta}_{\dot{\varepsilon}} \approx 125$ for the DP1200S. Furthermore, the quantitative evaluation of material similarity is carried out by Eq. (15b) in which $\kappa_{2}$ is assumed to 0.03 that being about one tenth of the maximum vertical coordinate of Fig. 6. In addition, the five orders of magnitude of characteristic strain-rate, $\hat{\dot{\varepsilon}}_{p}=10,100,200,400 \mathrm{~s}^{-1}$, are used to Eq. (16). Eight kinds of cases with the evaluation of material similarity as well as the basic scaling factors $\beta, \beta_{\rho}$ and $\beta_{V}$ are listed in Table 3. From $\hat{\dot{\varepsilon}}_{p}=10 \mathrm{~s}^{-1}$ to $\hat{\dot{\varepsilon}}_{p}=$ 
$400 \mathrm{~s}^{-1}$, the factor $\beta_{V}$ for the non-similitude material S355 changed by 38 percent; while, for the similitude material DP1200, it only changed by 1 percent.

Table 3 Evaluation of material similarity and three basic factors.

\begin{tabular}{llllllll}
\hline Scaled model & Material & $\tilde{\beta}_{\dot{\varepsilon}}$ & $\hat{\dot{\varepsilon}}_{m}\left[s^{-1}\right]$ & $|\Delta M n|$ & $\beta$ & $\beta_{\rho}$ & $\beta_{V}$ \\
\hline $1\left(\hat{\dot{\varepsilon}}_{p}=10 s^{-1}\right)$ & $\mathrm{S} 355$ & 78 & $0.78 \mathrm{E} 3$ & 0.107 & 0.01 & 1.019 & 0.949 \\
$2\left(\hat{\dot{\varepsilon}}_{p}=100 s^{-1}\right)$ & $\mathrm{S} 355$ & 78 & $0.78 \mathrm{E} 4$ & 0.159 & 0.01 & 1.019 & 1.137 \\
$3\left(\hat{\dot{\varepsilon}}_{p}=200 s^{-1}\right)$ & $\mathrm{S} 355$ & 78 & $1.56 \mathrm{E} 4$ & 0.169 & 0.01 & 1.019 & 1.219 \\
$4\left(\hat{\dot{\varepsilon}}_{p}=400 s^{-1}\right)$ & $\mathrm{S} 355$ & 78 & $3.12 \mathrm{E} 4$ & 0.176 & 0.01 & 1.019 & 1.313 \\
$5\left(\hat{\dot{\varepsilon}}_{p}=10 s^{-1}\right)$ & $\mathrm{DP} 1200$ & 125 & $1.25 \mathrm{E} 3$ & 0.006 & 0.01 & 1.0 & 1.315 \\
$6\left(\hat{\dot{\varepsilon}}_{p}=100 s^{-1}\right)$ & $\mathrm{DP} 1200$ & 125 & $1.25 \mathrm{E} 4$ & 0.010 & 0.01 & 1.0 & 1.314 \\
$7\left(\hat{\dot{\varepsilon}}_{p}=200 s^{-1}\right)$ & $\mathrm{DP} 1200$ & 125 & $2.50 \mathrm{E} 4$ & 0.019 & 0.01 & 1.0 & 1.308 \\
$8\left(\hat{\dot{\varepsilon}}_{p}=400 s^{-1}\right)$ & $\mathrm{DP} 1200$ & 125 & $5.00 \mathrm{E} 4$ & 0.030 & 0.01 & 1.0 & 1.297 \\
\hline
\end{tabular}

\section{- 3.2.2 Results}

The establishing method of numerical model of impacted circular plates is exactly the same as that in Section 3.1.2. The various responses of the center point and neutral surface of the circular plates are analyzed below.

The refined evaluation of similarity by the strain-rate and stress responses in time and space fields is plotted in Fig. 7. The responses of the non-similitude material S355 used for scaled model are very sensitive to the characteristic strain-rate $\hat{\dot{\varepsilon}}_{p}$ and have significant predicted errors, Fig. 7a, c, e and g. While, the best similitude material DP1200 responses are always nearly the same as the prototype responses, Fig. 7b, d, f and h. Some small degree of error in the local part can be attributed to the elasticity effects and the relaxation for $|\Delta M n|=0$. 

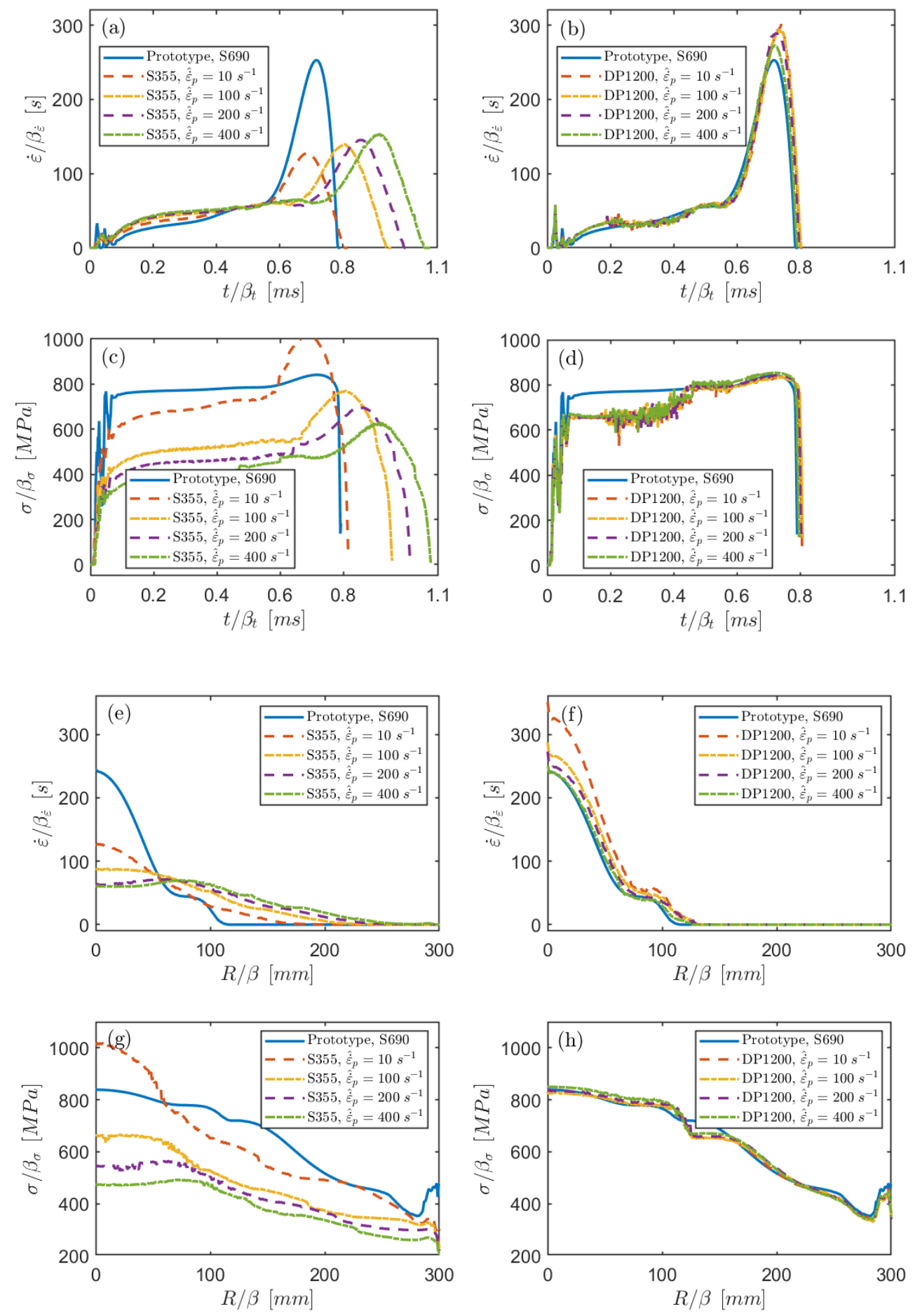

Fig. 7 Strain-rate and stress responses for scaled impacted circular plates with the similitude material S355 and the non-similitude material DP1200.

Further evaluation of similarity by structural displacement responses in time and space fields is shown in Fig. 8. The results show once more that the present method after using similitude material obtains direct and exact prediction. The maximum prediction error of the permanent displacement of the center point is also effectively 
reduced from 29 percent of the non-similitude material to the current 2 percent.
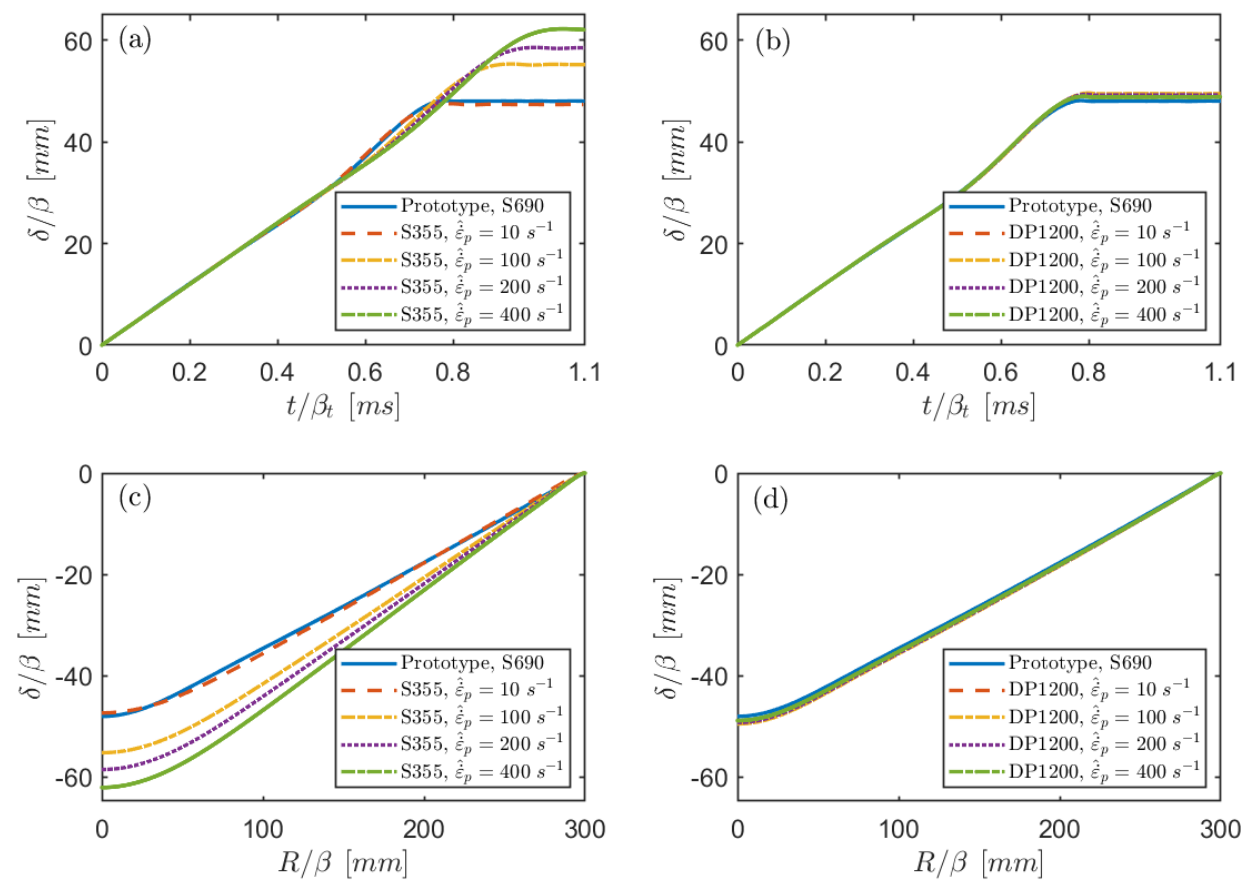

Fig. 8 Displacement responses for scaled impacted circular plates with the similitude material S355 and the non-similitude material DP1200.

\subsection{Impacted crooked plate with both strain hardening and strain-rate sensitive materials}

To verify material similarity of both the strain hardening effects and the strain-rate sensitive effects, a more complicated structure of two pre-bent plates (clamped together at both ends) with the initial crooked angle and subjected to an axial impact mass (as shown in Fig. 9) is used, and the Johnson-Cook equation (simplified)

$$
\sigma_{d}=\left(A+B \varepsilon^{\mathrm{n}}\right)\left(1+C \ln \left(\dot{\varepsilon} / \dot{\varepsilon}_{0}\right)\right)
$$

with material parameters $A, B, n, C$ and $\dot{\varepsilon}_{0}$ in Table. B.2 is considered. The structure is classified as 'type II' that being very sensitive to impact velocity [4]. 


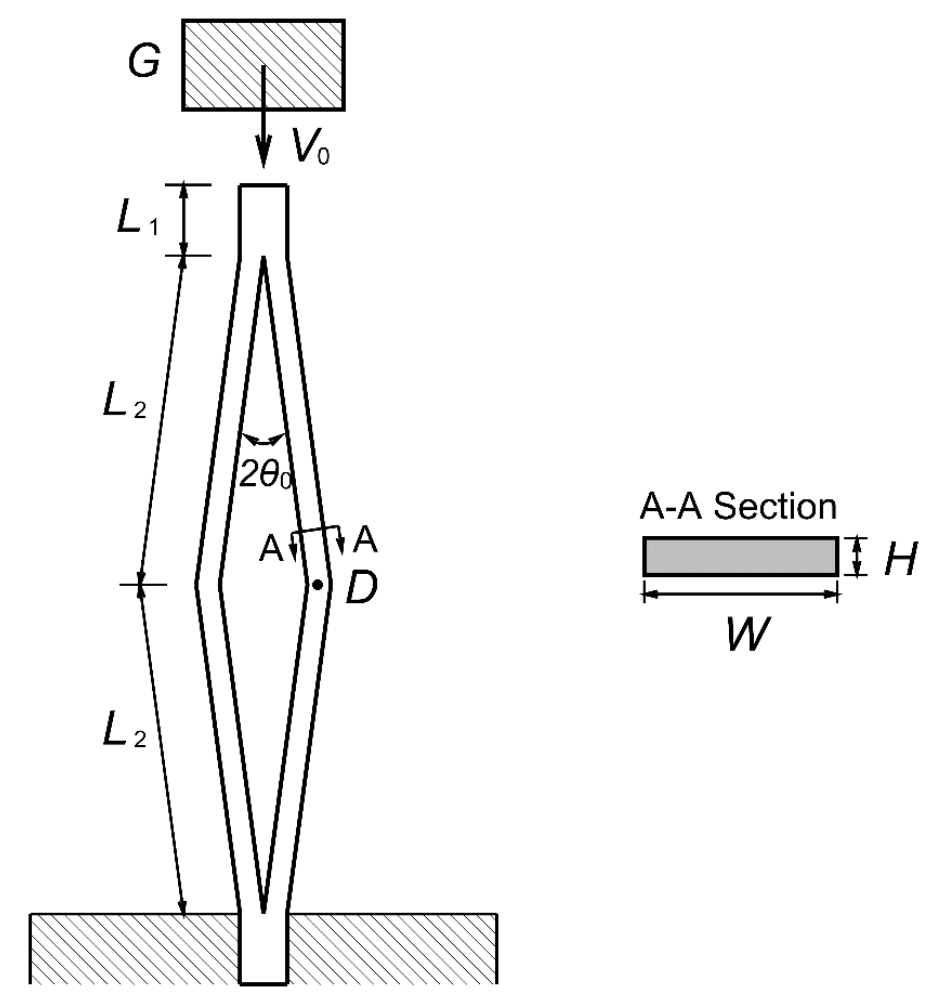

Fig. 9 Crooked plates under axial impact. $L_{1}=0.005 \mathrm{~m}, L_{2}=0.025 \mathrm{~m}, W=$ $0.051 \mathrm{~m}, H=0.0016 \mathrm{~m}, \theta_{0}=1.15^{\circ}, G_{p}=4 \mathrm{~kg}$ and $V_{0}=12 \mathrm{~m} / \mathrm{s}$.

The substitution of $E_{t}(\varepsilon, \dot{\varepsilon})=\partial \sigma_{d} / \partial \varepsilon=B n \varepsilon^{n-1}\left(1+C \ln \left(\dot{\varepsilon} / \dot{\varepsilon}_{0}\right)\right)$ into Eq. (9a) and the substitution of $K_{t}(\varepsilon, \dot{\varepsilon})=\partial \sigma_{d} / \partial \dot{\varepsilon}=\left(A+B \varepsilon^{n}\right)(C / \dot{\varepsilon})$ into Eq. (9b) obtain the material numbers

$$
\operatorname{Mn}\{\varepsilon\}=\frac{E_{t} \varepsilon}{\sigma_{d}}=n \frac{B \varepsilon^{n}}{A+B \varepsilon^{n}}
$$

and

$$
\operatorname{Mn}\{\dot{\varepsilon}\}=\frac{C}{1+C \ln \left(\dot{\varepsilon} / \dot{\varepsilon}_{0}\right)},
$$

respectively.

Then, the material similarity is evaluated by both 'material number - strain' curves (same with Fig. 3) and 'material number - strain' curves (as shown in Fig. 10) with eight different materials in Table B.2. Compared with Fig. 6, the $\operatorname{Mn}\{\dot{\varepsilon}\}$-values in Fig. 10 are small, which indicates that the strain-rate sensitivity of the Johnson-Cook equation is not very significant due to further considering strain hardening effects. 


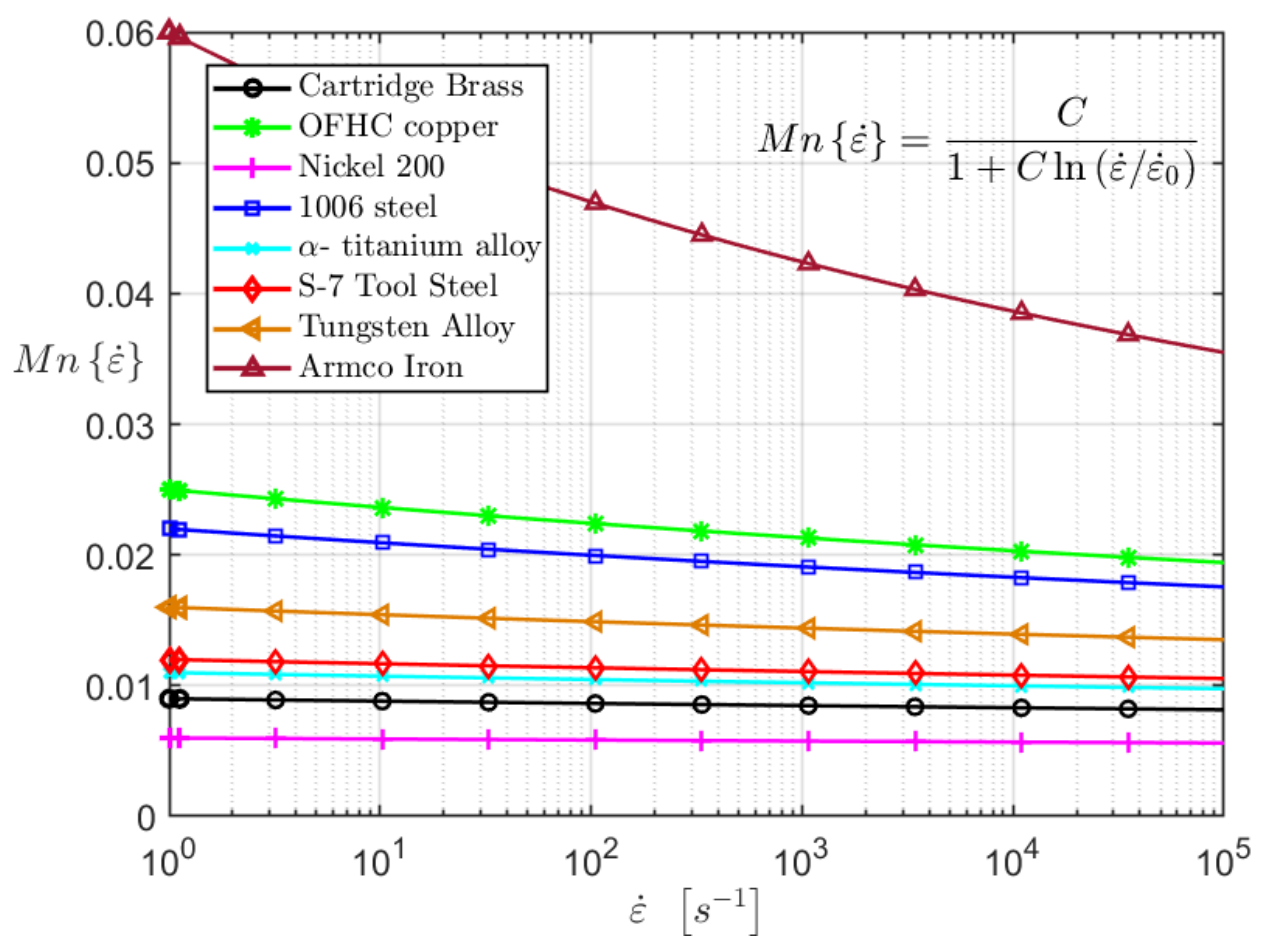

Fig. 10 Material similarity (only strain-rete effects) curves of the Johnson-Cook equation Eq. (21).

\section{- 3.3.1 Formulation}

For prototype, the geometry and loads are exactly the same as the impacted crooked plate. And the Tungsten Alloy is used. For scaled models, all geometric sizes are scaled by $\beta=1 / 10$. The impact mass and impact velocity are scaled by $\beta_{G}=$ $\beta_{\rho} \beta^{3}$ and $\beta_{V}$ (Eq. (16)), respectively. The best similitude material, S-7 Tool Steel (STS), and the non-similitude material (as a contrast), Armco Iron (AI), are selected intuitively, according to both Fig. 6 and Fig. 10. Furthermore, the quantitative evaluation for material similarity is carried out by both Eq. (15a) and Eq. (15b) in which $\kappa_{1}$ and $\kappa_{2}$ are assumed to be 0.03 . In addition, different orders of magnitude of the characteristic strain, $\hat{\varepsilon}=0.005,0.05,0.2$ and the characteristic strain rate, $\hat{\dot{\varepsilon}}_{p}=10$, $250,500 \mathrm{~s}^{-1}$ are used to Eq. (16). Ten kinds of loading cases with the evaluation of material similarity as well as the basic scaling factors $\beta, \beta_{\rho}$ and $\beta_{V}$ are listed in Table 4. From $\left(\hat{\varepsilon}_{p}=0.005, \hat{\dot{\varepsilon}}_{p}=10 s^{-1}\right)$ to $\left(\hat{\varepsilon}_{p}=0.2, \hat{\dot{\varepsilon}}_{p}=500 s^{-1}\right)$, the factor $\beta_{V}$ for the non-similitude material AI changed by 31 percent; while, for the similitude 
material STS, it only changed by 2 percent.

Table 4 Evaluation of material similarity and three basic factors.

\begin{tabular}{llllllllll}
\hline Scaled model & Material & $\beta_{\varepsilon}$ & $\hat{\varepsilon}_{m}$ & $|\Delta M n\{\varepsilon\}|$ & $\tilde{\beta}_{\dot{\varepsilon}}$ & $\hat{\dot{\varepsilon}}_{m}\left[s^{-1}\right]$ & $|\Delta M n\{\dot{\varepsilon}\}|$ & $\beta$ & $\beta_{V}$ \\
\hline $1\left(\hat{\varepsilon}_{p}=0.005, \hat{\dot{\varepsilon}}_{p}=10 s^{-1}\right)$ & AI & 1 & 0.005 & 0.084 & 5.00 & 50 & 0.033 & 0.1 & 0.630 \\
$2\left(\hat{\varepsilon}_{p}=0.005, \hat{\varepsilon}_{p}=500 s^{-1}\right)$ & AI & 1 & 0.005 & 0.084 & 5.00 & 2500 & 0.026 & 0.1 & 0.668 \\
$3\left(\hat{\varepsilon}_{p}=0.05, \hat{\varepsilon}_{p}=250 s^{-1}\right)$ & AI & 1 & 0.05 & 0.136 & 5.00 & 50 & 0.033 & 0.1 & 0.717 \\
$4\left(\hat{\varepsilon}_{p}=0.2, \hat{\varepsilon}_{p}=10 s^{-1}\right)$ & AI & 1 & 0.15 & 0.163 & 5.00 & 50 & 0.033 & 0.1 & 0.780 \\
$5\left(\hat{\varepsilon}_{p}=0.2, \hat{\varepsilon}_{p}=500 s^{-1}\right)$ & AI & 1 & 0.15 & 0.163 & 5.00 & 2500 & 0.026 & 0.1 & 0.826 \\
$6\left(\hat{\varepsilon}_{p}=0.005, \hat{\varepsilon}_{p}=10 s^{-1}\right)$ & STS & 1 & 0.005 & 0.012 & 14.97 & 150 & 0.004 & 0.1 & 1.555 \\
$7\left(\hat{\varepsilon}_{p}=0.005, \hat{\dot{\varepsilon}}_{p}=500 s^{-1}\right)$ & STS & 1 & 0.005 & 0.012 & 14.97 & 7486 & 0.004 & 0.1 & 1.543 \\
$8\left(\hat{\varepsilon}_{p}=0.05, \hat{\varepsilon}_{p}=250 s^{-1}\right)$ & STS & 1 & 0.05 & 0.019 & 14.97 & 7486 & 0.004 & 0.1 & 1.570 \\
$9\left(\hat{\varepsilon}_{p}=0.2, \hat{\varepsilon}_{p}=10 s^{-1}\right)$ & STS & 1 & 0.15 & 0.022 & 14.97 & 150 & 0.004 & 0.1 & 1.600 \\
$10\left(\hat{\varepsilon}_{p}=0.2, \hat{\varepsilon}_{p}=500 s^{-1}\right)$ & STS & 1 & 0.15 & 0.022 & 14.97 & 7486 & 0.004 & 0.1 & 1.588 \\
\hline
\end{tabular}

- 3.3.2 Results

The crooked plates were modelled in the ABAQUS software using the CPE4R elements and discretized with 25 elements in $L_{1}, 125$ elements in $L_{1}$ and 8 in thickness direction. The impact mass was modelled as a steel mass block with the Analytical Rigid. The contact between the impact mass and the plate used the Surface to Surface contact. The setting method of elasticity is the same as that in Section 3.1.2. The various responses of the impact mass, the point D (see Fig. 9) and the neutral surface of crooked plates are analyzed below.

The refined evaluation of similarity by the strain, strain-rate and stress responses in time and space fields is plotted in Fig. 11. The responses of the non-similitude material Iron used for scaled model are very sensitive to the characteristic strain $\hat{\varepsilon}_{p}$ and the characteristic strain-rate $\hat{\dot{\varepsilon}}_{p}$ and have significant predicted errors, and even failed prediction, Fig. 11a, c, e, g and 1. While, the best similitude material S-7 responses are always nearly the same as the prototype responses, Fig. 11b, d, f, h and m. Some small degree of error in the local part can be attributed to the elasticity effects and the relaxation for $|\Delta M n|=0$. 

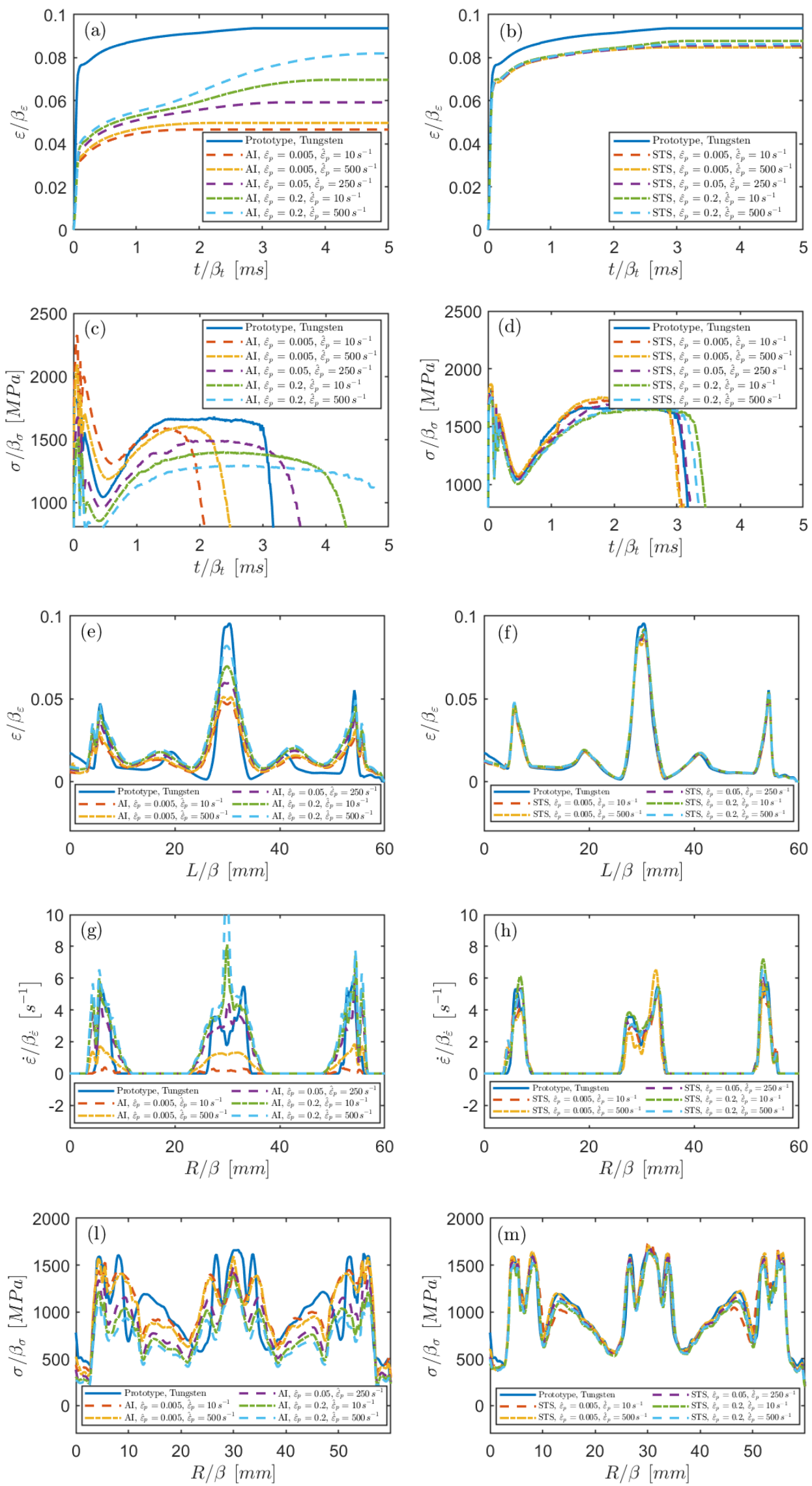

Fig. 11 Strain, strain-rate and stress responses for scaled impacted crooked plates 
with the similitude material (STS) and the non-similitude material (AI).

Further evaluation of similarity by structural displacement and force responses in time and space fields is shown in Fig. 12. The results show once more that the present method after using similitude material obtains direct and exact prediction when the strain hardening effects and strain-rate sensitive effects are coupled together. The maximum prediction error of the maximum displacement of the impact mass is also effectively reduced from 68 percent of the non-similitude material to the current 9 percent.
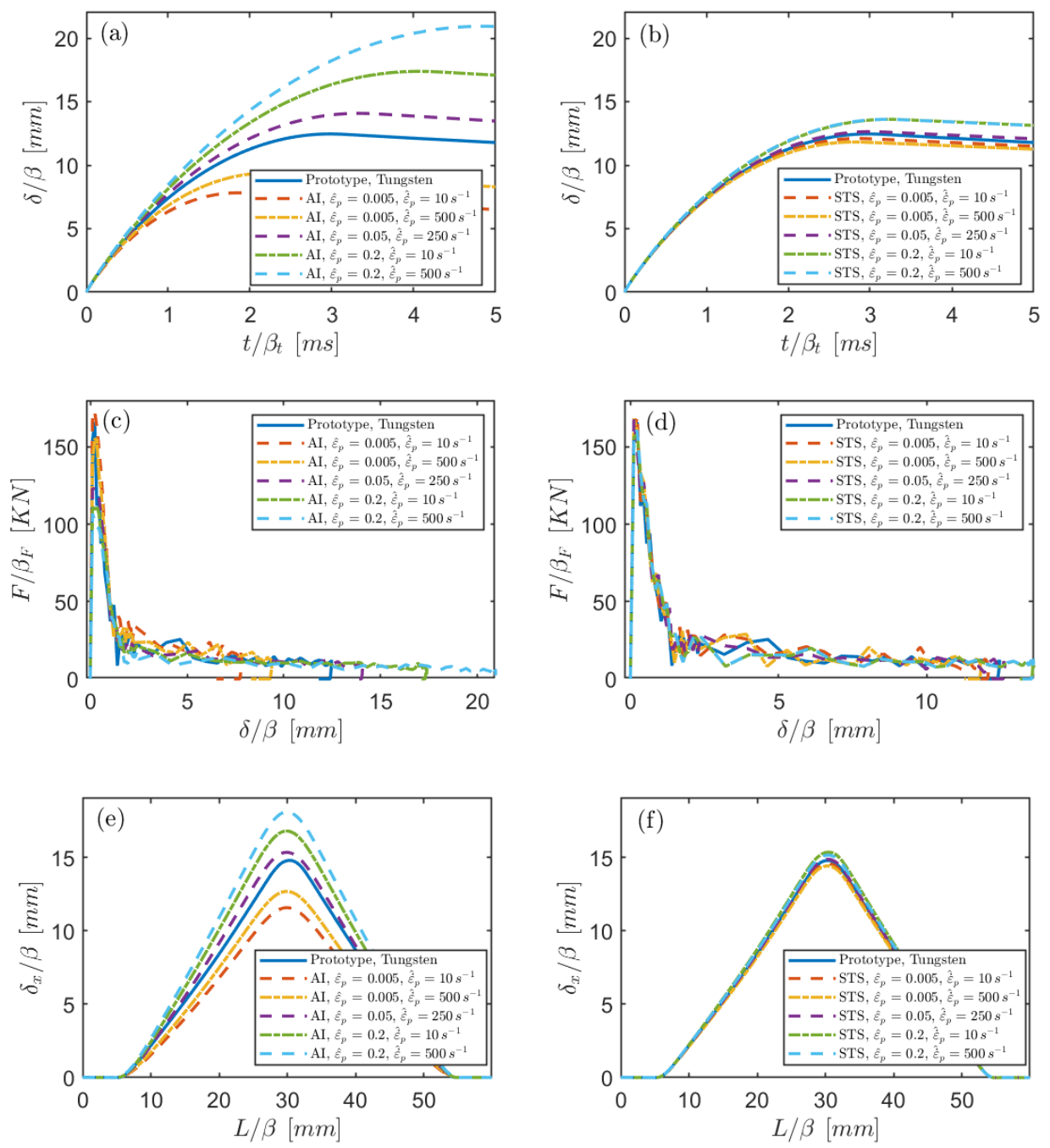

Fig. 12 The displacement and force response of impact mass and the horizontal displacement $\delta_{x}$ of neutral surface for scaled impacted crooked plates with the similitude material (STS) and the non-similitude material (AI). 


\section{Discussion}

\subsection{Extension of material similarity for elastic and temperature effects}

Is the method suggested in this paper instructive for similarity of elastic and thermal effects of solid materials? The answer is positive.

- When the linear elastic constitutive model $\sigma=E \varepsilon^{e}$ is used, the material number is expressed as

$$
\operatorname{Mn}\left\{\varepsilon^{e}\right\}=\frac{E \varepsilon^{e}}{\sigma} \equiv 1
$$

where $E$ and $\varepsilon^{e}$ are Young's modulus and elastic strain, respectively.

It immediately asserts that all linear elastic materials are always similar (if influence of Poisson's ratio for similarity can be ignored) since $M n_{m}\left\{\varepsilon^{e}\right\} \equiv M n_{p}\left\{\varepsilon^{e}\right\}$. Thus, the use of $\beta_{\sigma}=\beta_{\rho} \beta_{V}^{2}$ and $\beta_{\varepsilon}=1$ (Table 1) for $\sigma=E \varepsilon^{e}$ can obtain the direct and exact correction factor of velocity as $\beta_{V}=\sqrt{\beta_{E} / \beta_{\rho}}$.

- When the Johnson-Cook equation with the temperature effects (see Table B.2) is used, the material number is expressed as

$$
M n\{T\}=\frac{\left[\partial \sigma_{d}(\varepsilon, \dot{\varepsilon}, T) / \partial T\right] T}{\sigma_{d}}=m \frac{T^{*}}{1-T^{*}} \frac{T}{T-T_{0}} .
$$

The temperature effect similarity of eight different materials are further depicted in Fig. 13 where $T_{0}$ is assumed to be the room temperature $298.15 \mathrm{~K}$. Apparently, if $\beta_{T}=1$, the 1006 steel, the $\alpha$ - titanium alloy and the S-7 Tool Steel can be intuitively considered as the best similitude materials for the Tungsten Alloy. For 1006 steel, the ratio of the temperature effects $\left(1-T_{m}^{*}\right) /\left(1-T_{p}^{*}\right)$ is 1.00 and 1.03 when $T_{p}=$ $300 \mathrm{~K}$ and $800 \mathrm{~K}$, respectively. Because it is almost unchanged, the direct and exact property is verified. As a contrast, however, the ratio for Armco Iron (consider as nonsimilitude material) are 0.98 and 0.70 , respectively. It changes significantly. 


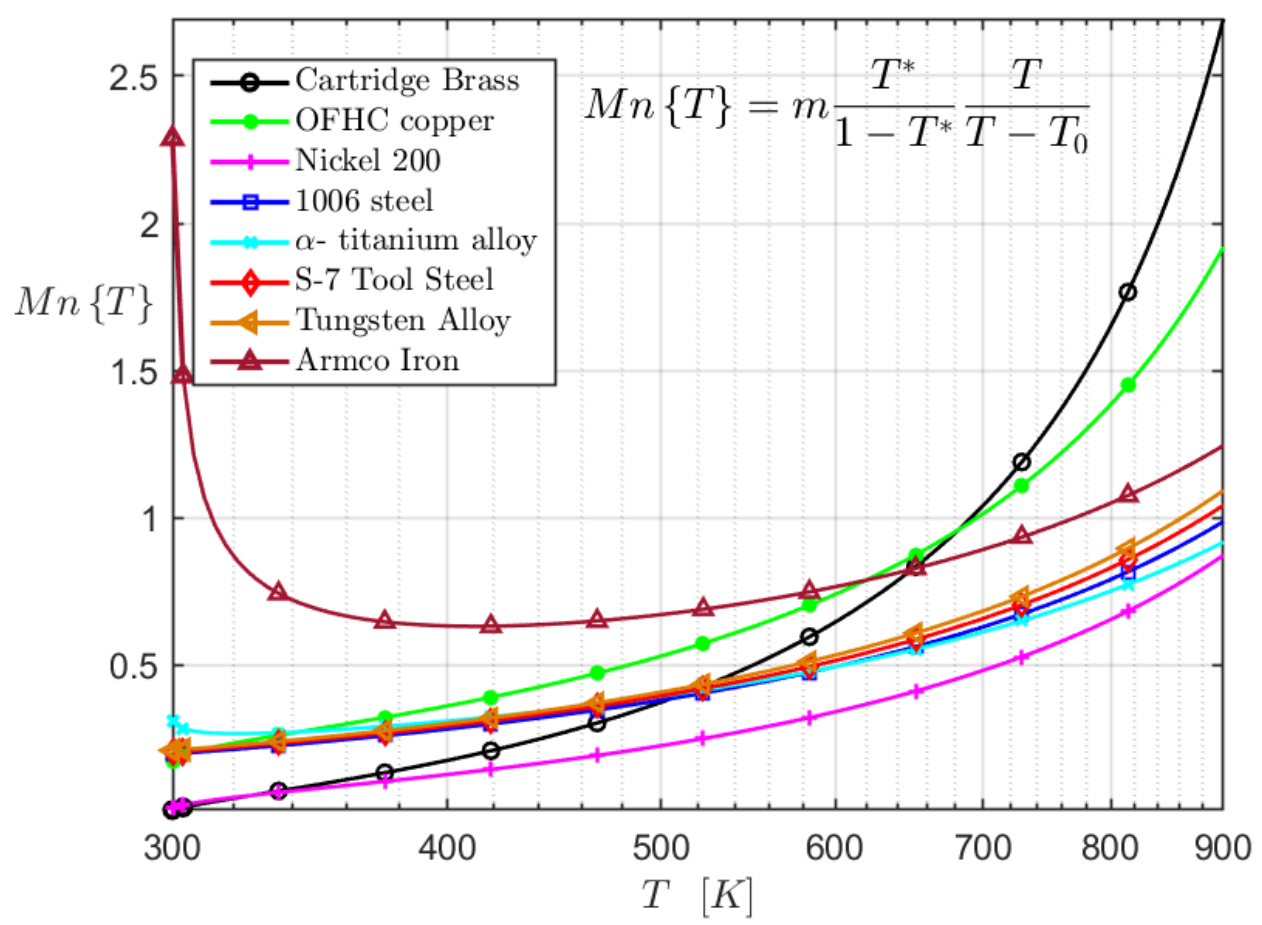

Fig. 13 Material similarity curves of the temperature effect of the Johnson-Cook equation.

- In summary, for similarity of arbitrary material effects, the material number $M n$ can be generalized to the form

$$
\operatorname{Mn}\left\{X_{i}\right\}=\frac{\left[\partial f\left(X_{1}, \ldots, X_{i}, \ldots\right) / \partial X_{i}\right] X_{i}}{f\left(X_{1}, \ldots, X_{i}, \ldots\right)}=\frac{\partial \ln f\left(X_{1}, \ldots, X_{i}, \ldots\right)}{\partial \ln X_{i}}
$$

for the general constitutive model $\sigma=f\left(X_{1}, X_{i}, \ldots\right)$ with $i$ mechanical variables. The form $\partial \ln ($ funtion)/ $\ln ($ independent variable) is an important operator with dimensionless property.

\section{Conclusion}

This paper proposed a material similarity framework for strain hardening and strain-rate sensitive solid materials, and solves the inherent non-direct and inaccuracy difficulty existing in all previous similarity theory of structural impact when using the basic correction factors. Since the proposed material similarity is an important property for the most basic stress - strain/strain-rate relation of materials, it must be respected in solid scaling experiments. Compared with previous works about scaling that only focus on the correction of basic scaling factors, the new proposed method is mainly reflected 
in five aspects.

(1) Based on the similarity analysis of the viscous-plasticity constitutive equation, the material dimensionless numbers of strain hardening and strain-rate sensitivity are derived, which are the intrinsic requirement of similarity theory for material basic mechanical properties.

(2) 'Material number - strain/strain-rate' function curves are further proposed to evaluate material similarity between scale model and prototype. According to this curve, it is very intuitive and convenient to select the best similitude materials for the scaled model when carrying out the scaled solid experiments.

(3) The direct and accurate solution of the basic correction factors in similarity theory is proved theoretically. Since this solution is independent of the structural strain and strain-rate responses, the previous inherent non-direct and inaccuracy difficulty is overcome radically.

(4) By using the best similitude material to scaled model and prototype, different materials with strain hardening and strain-rate sensitive effects are allowed to precisely similar in various behaviors of the impacted circular plate and the more complicated impacted crooked plate in time and space fields. However, when using the nonsimilitude material, the similarity errors are not controllable, which often leads to failed prediction.

(5) The proposed method is not only suitable for similarity of material strain hardening and strain-rate sensitive effects, but also effective for similarity of other effects of materials such as elastic and temperature.

Since the material similarity is an important basic mechanical property of solid materials, it is necessary to be further explored in the future.

\section{Appendix A}

The direct and exact solution Eq. (14) is verified as follows.

Consider the Norton-Hoff equation [16,26]

$$
\sigma_{d}=\sigma_{0} \varepsilon^{n_{1}}\left(\dot{\varepsilon} / \dot{\varepsilon}_{0}\right)^{n_{2}}
$$


where $\dot{\varepsilon}_{0}$ is the corresponding strain rate for $\sigma_{0} ; n_{1}$ and $n_{2}$ are material constants.

The material numbers Eq. (9a) and Eq. (9b) are derived as

$$
\operatorname{Mn}\{\varepsilon\}=\frac{E_{t} \varepsilon}{\sigma_{d}}=n_{1}
$$

and

$$
\operatorname{Mn}\{\dot{\varepsilon}\}=\frac{K_{t} \dot{\varepsilon}}{\sigma_{d}}=n_{2}
$$

respectively.

If $\left(n_{1}\right)_{m}=\left(n_{1}\right)_{p}$ and $\left(n_{2}\right)_{m}=\left(n_{2}\right)_{p}$, we obtain

$$
\beta_{\sigma_{d}}=\beta_{\sigma_{0}} \frac{\left(\varepsilon_{m}\right)^{n_{1}}\left(\dot{\varepsilon}_{m} /\left(\dot{\varepsilon}_{0}\right)_{m}\right)^{n_{2}}}{\left(\varepsilon_{p}\right)^{n_{1}}\left(\dot{\varepsilon}_{p} /\left(\dot{\varepsilon}_{0}\right)_{p}\right)^{n_{2}}}=\beta_{\sigma_{0}} \beta_{\varepsilon}^{n_{1}}\left(\beta_{\dot{\varepsilon}} / \beta_{\dot{\varepsilon}_{0}}\right)^{n_{2}} \equiv \text { constant }
$$

The substitution of $\beta_{\sigma_{d}}=\beta_{\rho} \beta_{V}^{2}, \beta_{\varepsilon}=1, \beta_{\dot{\varepsilon}}=\beta_{V} / \beta$ (Table 1) and Eq. (A.3) into Eq. (3) obtains the direct and exact solution as

$$
\beta_{V}=\sqrt[\left(2-n_{2}\right)]{\frac{\beta_{\sigma_{0}}}{\beta_{\rho}}\left(\frac{1}{\beta \beta_{\dot{\varepsilon}_{0}}}\right)^{n_{2}}} \equiv \mathrm{constant} \text { if }\left\{\begin{array}{l}
\left(n_{1}\right)_{m}=\left(n_{1}\right)_{p} \\
\left(n_{2}\right)_{m}=\left(n_{2}\right)_{p}
\end{array}\right.
$$

which has nothing to do with the structural strain and the strain-rate responses.

\section{Appendix B}

The material parameters used in Section 3 are as follows.

Table B.1 Material properties of the Johnson-Cook constitutive equation [29] $\sigma_{d}=$ $\left(A+B \varepsilon^{n}\right)\left(1+C \ln \dot{\varepsilon}^{*}\right)\left(1-T^{* m}\right)$, where $A$ is quasi-static flow stress; $B, n, C$ and $m$ are material constants; $\dot{\varepsilon}^{*}=\dot{\varepsilon} / \dot{\varepsilon}_{0}$ with $\dot{\varepsilon}_{0}$ being reference strain-rate; $T^{*}=$ $\left(T-T_{0}\right) /\left(T_{m}-T_{0}\right)$ with $T, T_{0}$ and $T_{\mathrm{m}}$ being temperature, reference temperature and melting point, respectively. Note: * from [31]; ** from [32].

\begin{tabular}{lllllllllll}
\hline Material & Density & Elastic & Poisson's & $T_{\mathrm{m}}$ & $\mathrm{A}$ & $\mathrm{B}$ & $\mathrm{n}$ & $\mathrm{C}$ & $\dot{\varepsilon}_{0}$ & $\mathrm{~m}$ \\
& {$\left[\mathrm{~kg} / \mathrm{m}^{3}\right]$} & modulus & Ratio & {$[\mathrm{K}]$} & {$[\mathrm{MPa}]$} & {$[\mathrm{MPa}]$} & & & {$\left[\mathrm{s}^{-1}\right]$} \\
& & {$[\mathrm{GPa}]$} & & & & & & & & \\
& & & & & & & & & & \\
\hline Cartridge Brass [29] & $8.52 \mathrm{E} 3$ & $110 *$ & $0.35^{*}$ & 1189 & 112 & 505 & 0.42 & 0.009 & 1 & 1.68 \\
\hline
\end{tabular}




\begin{tabular}{lcccccccccccc}
\hline OFHC copper [29] & $8.96 \mathrm{E} 3$ & $115 *$ & $0.33^{*}$ & 1356 & 90 & 292 & 0.31 & 0.025 & 1 & 1.09 \\
Nickel 200 [29] & $8.90 \mathrm{E} 3$ & $204 *$ & $0.31^{*}$ & 1725 & 163 & 648 & 0.33 & 0.006 & 1 & 1.44 \\
1006 steel [29] & $7.89 \mathrm{E} 3$ & $207 *$ & $0.30^{*}$ & 1811 & 350 & 275 & 0.36 & 0.022 & 1 & 1.00 \\
$\alpha$ - titanium alloy [30] & $4.51 \mathrm{E} 3 *$ & $103 *$ & $0.34^{*}$ & $1933 *$ & 837 & 731 & 0.31 & 0.011 & 1 & 0.91 \\
S-7 Tool Steel [29] & $7.75 \mathrm{E} 3$ & $207 *$ & $0.30^{*}$ & 1763 & 1539 & 477 & 0.18 & 0.012 & 1 & 1.00 \\
Tungsten Alloy [29] & $17.0 \mathrm{E} 3$ & $400 *$ & $0.28^{*}$ & 1723 & 1506 & 177 & 0.12 & 0.016 & 1 & 1.00 \\
Armco Iron [29] & $7.89 \mathrm{E} 3$ & $207 *$ & $0.30 *$ & 1811 & 175 & 380 & 0.32 & 0.06 & 1 & 0.55 \\
\hline
\end{tabular}

Table B.2 Material properties of the Cowper-Symonds constitutive equation [3] $\sigma_{d}=$ $\sigma_{0}\left[1+(\dot{\varepsilon} / D)^{1 / P}\right]$ with $D$ and $P$ being the material constants. Note: * from [31].

\begin{tabular}{lllllll}
\hline Material & Density & Elastic & Poisson's & $\sigma_{0}$ & D & P \\
& {$\left[10^{3} \mathrm{~kg} / \mathrm{m}^{3}\right]$} & Modulus [GPa] & Ratio & {$[\mathrm{MPa}]$} & {$[\mathrm{s}-1]$} & \\
\hline S355 steel [33] & $8.00 *$ & $193 *$ & $0.30 *$ & 448 & 4945 & 2.70 \\
High-strength steel S690 [34] & $7.85 *$ & $207 *$ & $0.30 *$ & 722 & 18404 & 2.38 \\
Mild steel [3] & $7.85 *$ & $207 *$ & $0.30 *$ & $210 *$ & 40.4 & 5 \\
Dual Phase 1200 steel [35] & $7.85 *$ & $207 *$ & $0.30 *$ & 1130 & 55063631 & 5.63 \\
Stainless steel 304 [3] & $8.00 *$ & $193 *$ & $0.30 *$ & $205 *$ & 100 & 10 \\
Aluminum 6061-T6 [3] & $2.70 *$ & $69 *$ & $0.33 *$ & $276 *$ & 6500 & 4 \\
Copper [36] & $8.89 *$ & $115 *$ & $0.33 *$ & 205 & 1778 & 4.99 \\
$\alpha$ - titanium Ti-50A [3] & $4.51 *$ & $103 *$ & $0.34 *$ & $170 *$ & 120 & 9 \\
\hline
\end{tabular}

\section{Authorship roles}

Shuai Wang: Conceptualization, Methodology, Formal analysis, Writing; Fei Xu: Conceptualization, Supervision, Resources, Funding acquisition; Xiaoyu Zhang: Investigation, Software. Leifeng Yang: Validation.

\section{Declaration of Competing Interest}

The authors declare that they have no known competing financial interests or personal relationships that could have appeared to influence the work reported in this paper. 


\section{References:}

[1] Coutinho, C.P., Baptista, A.J., Rodrigues, J.D., 2016. Reduced scale models based on similitude theory: A review up to 2015. Engineering Struct., 119, 81-94. http://dx.doi.org/10.1016/j.engstruct.2016.04.016

[2] Casaburo, A., Petrone, P., Franco, F., Rosa, S.D., 2019. A Review of Similitude Methods for Structural Engineering. Appl. Mech. Rev., 71(3): 030802. https://doi.org/10.1115/1.4043787

[3] Jones, N., 1989. Structural impact. Cambridge University Press, Cambridge.

[4] Lu, G., Yu, T.X., 2003. Energy absorption of structures and materials. Cambridge Woodhead Publishing, Cambridge.

[5] Booth, E., Collier, D., Miles, J., 1983. Impact scalability of plated steel structures. In: Jones, N., Wierzbicki, T. (Eds.), Structural Crashworthiness, Butterworks, London, pp. 136-174.

[6] Jones, N., Jouri, W. and Birch, R., 1984. On the scaling of ship collision damage, International Maritime Association of the East Mediterranean, Third International Congress on Marine Technology, Athens, 2, 287-94.

[7] Jiang, P., Wang, W., Zhang, G.J., 2006. Size effects in the axial tearing of circular tubes during quasi-static and impact loadings. Int. J. Impact Eng. 32, 2048-2065. https://doi.org/10.1016/j.ijimpeng.2005.07.001

[8] Fu, S., Gao, X., Chen, X., 2018. The similarity law and its verification of cylindrical lattice shell model under internal explosion. Int. J. Impact Eng. 122, 38-49. https://doi.org/10.1016/j.ijimpeng.2018.08.010

[9] Hu, Y.Q, Zhao, Y.P., 2001. Scale effect of plastic strain rate. Chinese journal of aeronautics, 14, 37-43.

[10] Drazetic, P., Ravalard, Y., Dacheux, F., Marguet, B., 1994. Applying non-direct similitude technique to the dynamic bending collapse of rectangular section tubes. Int. J. Impact Eng. 15, 797-814. https://doi.org/10.1016/0734-743X(94)90066-T

[11] Oshiro, R.E., Alves, M., 2004. Scaling impacted structures. Arch. Appl. Mech. 74, 
130-145. https://doi.org/10.1007/s00419-004-0343-8

[12] Oshiro, R.E., Alves, M., 2007. Scaling of cylindrical shells under axial impact. Int. J. Impact Eng. 34, 89-103. https://doi.org/10.1016/j.ijimpeng.2006.02.003

[13] Alves, M., Oshiro, R.E., 2006. Scaling the impact of a mass on a structure. Int. J. Impact Eng. 32, 1158-1173. https://doi.org/10.1016/j.ijimpeng.2004.09.009

[14] Jiang, Z.R., Zhong, Y.K., Shi K.R., Luo, B., 2016. Gravity-based impact comparability rule of single-layer reticulated shells and its numerical verification. J. South China Univ. Techno. (Nat. Sci. Ed.) 44: 43-48. (in Chinese) https://doi.org/10.3969/j.issn.1000-565X.2016.10.007

[15] Wei, D., Hu, C., 2019. Scaling of an impacted reticulated dome using partial similitude method. Lat. Am. J. Solids Struct. 16, e158. https://doi.org/10.1590/1679$\underline{78255342}$

[16] Oshiro, R.E., Alves, M., 2009. Scaling of structures subject to impact loads when using a power law constitutive equation. Int. J. Solids Struct. 46, 3412-3421. https://doi.org/10.1016/j.ijsolstr.2009.05.014

[17] Alves, M., Oshiro, R.E., 2006. Scaling impacted structures when the prototype and the model are made of different materials. Int. J. Solids Struct. 43, 2744-2760. https://doi.org/10.1016/j.ijsolstr.2005.03.003

[18] Mazzariol, L.M., Alves, M., 2013. Experimental study on scaling of circular tubes subjected to dynamic axial crushing using models of different materials. Proceedings of the 22nd international congress of mechanical engineering (COBEM 2013).

[19] Mazzariol, L.M., Oshiro, R.E., Alves, M., 2016. A method to represent impacted structures using scaled models made of different materials. Int. J. Impact Eng. 90, 8194. https://doi.org/10.1016/j.ijimpeng.2015.11.018

[20] Mazzariol, L.M., Alves, M., 2019. Similarity laws of structures under impact load: Geometric and material distortion. Int. J. Mech. Sci. 157-158, 633-647. https://doi.org/10.1016/j.ijmecsci.2019.05.011

[21] Sadeghi, H., Davey, K., Darvizeh, R., Darvizeh, A., 2019. A scaled framework for 
strain rate sensitive structures subjected to high rate impact loading. Int. J. Impact Eng. 125, 229-245. https://doi.org/10.1016/j.ijimpeng.2018.11.008

[22] Sadeghi, H., Davey, K., Darvizeh, R., Darvizeh, A., 2019. Scaled models for failure under impact loading. Int. J. Impact Eng. 129, 36-56. https://doi.org/10.1016/j.ijimpeng.2019.02.010

[23] Wang, S., Xu, F., Dai, Z., 2020. Suggestion of the DLV dimensionless number system to represent the scaled behavior of structures under impact loads. Arch. Appl. Mech. 90, 707-719. https://doi.org/10.1007/s00419-019-01635-9

[24] Wang, S., Xu, F., Zhang, X., Dai, Z., 2020. Suggestion of a framework of similarity laws for geometric distorted structures subjected to impact loading. Preprints 2020, 2020020394. https://doi.org/10.20944/preprints202002.0394.v1

[25] Wang, S. Xu, F., Dai, Z., et al.., 2020. A direct scaling method for the distortion problems of structural impact. Chinese Journal of Theoretical and Applied Mechanics. 52, 774-786 (in Chinese)). https://doi.org/10.6052/0459-1879-19-327

[26] Davey, K., Bylya, O., Krishnamurthy, B., 2020. Exact and inexact scaled models for hot forging. Int. J. Solids Struct., 203, 110-130. https://doi.org/10.1016/j.ijsolstr.2020.06.024

[27] Zhao, Y.P., Liu, S., 2001. On the definition of coefficient of strain-rate sensitivity. Chinese journal of aeronautics. 14, 78-82. http://www.hkxb.net.cn/cja/2001/01/0037/ [28] Meyers, M.A., Chawla, K.K., 2009. Mechanical behavior of materials. Cambridge University Press, New York.

[29] Johnson, R., Cook, W.K., 1983. A constitutive model and data for metals subjected to large strains high strain rates and high temperatures. The 7th International Symposium on Ballistics. The Hague, 541-547.

[30] Bobbili, R., Madhu, V., 2018. Crystal plasticity modeling of a near alpha titanium alloy under dynamic compression. Journal of Alloys and Compounds, 759, 85-92. https://doi.org/10.1016/j.jallcom.2018.05.167

[31] William, D., Callister, Jr., 2007. Materials science and engineering: an introduction. John Wiley \& Sons.

[32] Khan A.S., Suh Y.S., Kazmi R., 2004. Quasi-static and dynamic loading responses 
and constitutive modeling of titanium alloys. Int. J. Plasticity 20, 2233-2248. https://doi.org/10.1016/j.ijplas.2003.06.005

[33] Cadoni, E., Forni, D., Gieleta, R., Kruszka, L., 2018. Tensile and compressive behaviour of S355 mild steel in a wide range of strain rates. Eur. Phys. J. Special Topics 227, 29-43. https://doi.org/10.1140/epjst/e2018-00113-4

[34] Yang, X., Yang, H., Zhang, S., Rate-dependent constitutive models of S690 highstrength structural steel, Construction and Building Materials, 198, 597-607. https://doi.org/10.1016/j.conbuildmat.2018.11.285

[35] Singh, N.K., Cadoni, E., Singha, M.K., Gupta, N.K., 2011. Mechanical behavior of advanced high strength steel at high strain rates. Applied Mechanics and Materials, 82, 178-183. https://doi.org/10.4028/www.scientific.net/AMM.82.178

[36] Vesenjak, M., Hokamoto, K., Anzel, I., Sato, A., Tsunoda, R., Krstulovi-Opara, L., Ren, Z., 2015. Influence of the explosive treatment on the mechanical properties and microstructure of copper. Materials and Design, 75, 85-94. http://dx.doi.org/10.1016/j.matdes.2015.03.026 